Article

\title{
Synthesis of 1,4-Bis(phenylethynyl)benzenes and Their Application as Blue Phase Liquid Crystal Composition
}

\author{
Ning $\mathrm{Li}^{2,3}$, Zhengqiang $\mathrm{Li}^{2,3}$, Xing Zhang ${ }^{2,3}$ and Ruimao Hua ${ }^{1,2,3, *}$ \\ 1 Department of Chemistry, Tsinghua University, Beijing 100084, China \\ 2 Beijing R\&D Center, Shijiazhuang Chengzhi Yonghua Display Materials Co. Ltd., \\ Beijing 100083, China; E-Mails: nn13875@163.com (N.L.); \\ zhengqiang9527@gmail.com (Z.L.); zx@ @lichem.com (X.Z.) \\ 3 Hebei Engineering \& Technology Center for FPD Materials, Shijiazhuang 050091, China \\ * Author to whom correspondence should be addressed; E-Mail: ruimao@ mail.tsinghua.edu.cn; \\ Tel.: +86-10-6279-2596; Fax: +86-10-6277-1149.
}

Received: 14 September 2013; in revised form: 16 October 2013 / Accepted: 11 November 2013 / Published: 25 November 2013

\begin{abstract}
A number of 1,4-bis(phenylethynyl)benzene derivatives (BPEBs) and their analogues with different numbers of side-substitute fluorine atoms on benzene rings, and alkyl chains, ethoxyl groups, fluorine atoms and trifluoromethyl groups as the end groups have been synthesized. The effects of the different substituents on their properties such as thermal behavior of melting point and clearing point, the temperature of nematic phase, optical anisotropy and dielectric anisotropy have been well investigated, and it has been found that some BPEBs have a wide range of the nematic phase temperature with high optical anisotropy $(\Delta n)$ and acceptable dielectric anisotropy $(\Delta \varepsilon)$, which have been applied as the crucial compositions to constitute a liquid crystal mixture having the properties of $\Delta \varepsilon=29.0$ and $\Delta n=0.283$ at $25{ }^{\circ} \mathrm{C}$. With the addition of the chiral dopant to the obtained liquid crystal mixture, blue phase liquid crystal with a blue phase temperature range of $8^{\circ} \mathrm{C}$ has been achieved.
\end{abstract}

Keywords: 1,4-bis(phenylethynyl)benzene derivatives; blue phase; liquid crystal 


\section{Introduction}

Liquid crystals (LCs) have had a multitude of applications in the past few decades [1,2]. One of the important and unique applications is their use as the key fundamental materials to develop LC displays [3], which have actually changed people's lifestyle due to the use of mobiles, notebook computers, flat panel desktop monitors, and LCD televisions, etc. The development of excellent LC displays, which have the advantages of fast response, high contrast ratio, and low driving voltage, depends greatly on the development of new types and properties of LCs. The demand for LCDs with a fast response is one of the crucial factors to improve the quality of the displays, and blue phase liquid crystal (BPLC) is commonly considered to be one of the strongest candidates [4-7].

On the other hand, the molecular and electronic structures of 1,4-bis(phenylethynyl)-benzene derivatives (BPEBs), as well as their applications have attracted much attention recently [8-12]. In particular, BPEBs have been applied as the important components in LCs with the characters of high melting point (mp), clearing point (cp), and large optical anisotropy values $(\Delta n)$ [13-22]. It is important and interesting to investigate the substituent effect on the properties of BPEBs. Therefore, in this paper, we describe the synthesis of a number of BPEBs with different alkyl chains, the numbers of fluorine atoms and other substituents in benzene rings as shown in Scheme 1, and the study on the substituent effects on their properties, as well as their application as blue phase liquid crystal composition.

Scheme 1. The structures of 1,4-bis(phenylethynyl)benzene derivatives and analogues (1-25).
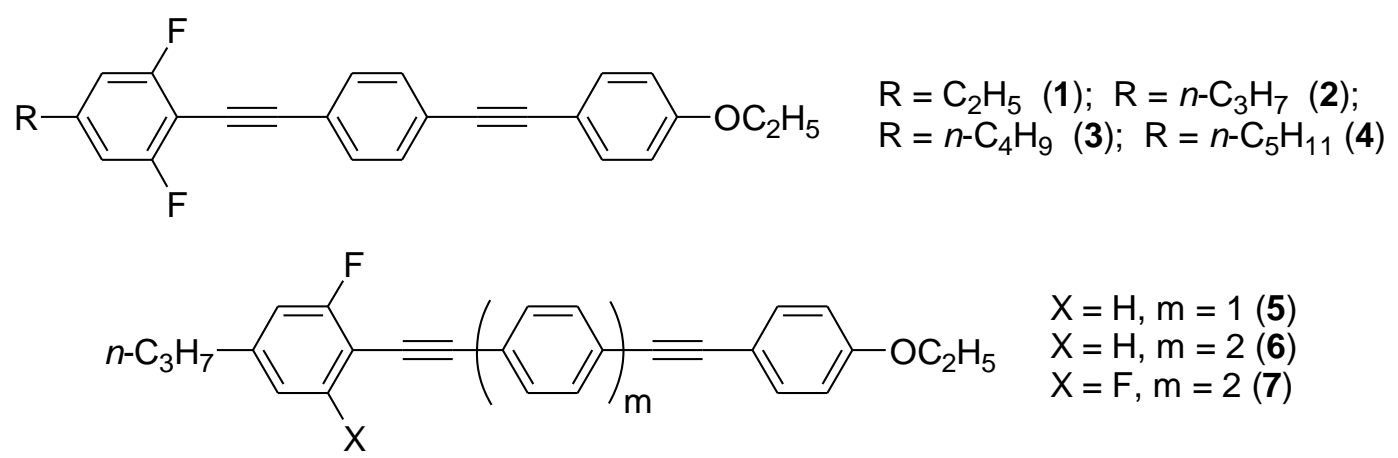

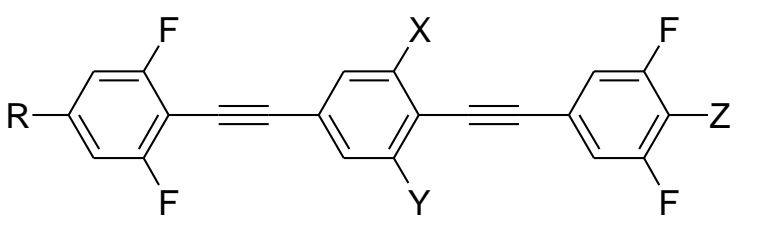

$$
\begin{aligned}
& \mathrm{R}=n-\mathrm{C}_{4} \mathrm{H}_{9}, \mathrm{X}=\mathrm{Y}=\mathrm{H}, \mathrm{Z}=\mathrm{OCF}_{3}(14) \\
& \mathrm{R}=n-\mathrm{C}_{4} \mathrm{H}_{9}, \mathrm{X}=\mathrm{Y}=\mathrm{H}, \mathrm{Z}=\mathrm{F}(15) \\
& \mathrm{R}=n-\mathrm{C}_{4} \mathrm{H}_{9}, \mathrm{X}=\mathrm{F}, \mathrm{Y}=\mathrm{H}, \mathrm{Z}=\mathrm{OCF}_{3}(16) \\
& \mathrm{R}=n-\mathrm{C}_{4} \mathrm{H}_{9}, \mathrm{X}=\mathrm{Z}=\mathrm{F}, \mathrm{Y}=\mathrm{H}(17) \\
& \mathrm{R}=n-\mathrm{C}_{4} \mathrm{H}_{9}, \mathrm{X}=\mathrm{Y}=\mathrm{F}, \mathrm{Z}=\mathrm{OCF}_{3}(18) \\
& \mathrm{R}=n-\mathrm{C}_{4} \mathrm{H}_{9}, \mathrm{X}=\mathrm{Y}=\mathrm{Z}=\mathrm{F}(19)
\end{aligned}
$$

$$
\begin{aligned}
& \mathrm{R}=n-\mathrm{C}_{3} \mathrm{H}_{7}, \mathrm{X}=\mathrm{Y}=\mathrm{H}, \mathrm{Z}=\mathrm{OCF}_{3}(\mathbf{8}) \\
& \mathrm{R}=n-\mathrm{C}_{3} \mathrm{H}_{7}, \mathrm{X}=\mathrm{Y}=\mathrm{H}, \mathrm{Z}=\mathrm{F}(\mathbf{9}) \\
& \mathrm{R}=n-\mathrm{C}_{3} \mathrm{H}_{7}, \mathrm{X}=\mathrm{F}, \mathrm{Y}=\mathrm{H}, \mathrm{Z}=\mathrm{OCF}_{3}(\mathbf{1 0}) \\
& \mathrm{R}=n-\mathrm{C}_{3} \mathrm{H}_{7}, \mathrm{X}=\mathrm{Z}=\mathrm{F}, \mathrm{Y}=\mathrm{H}(\mathbf{1 1}) \\
& \mathrm{R}=n-\mathrm{C}_{3} \mathrm{H}_{7}, \mathrm{X}=\mathrm{Y}=\mathrm{F}, \mathrm{Z}=\mathrm{OCF}_{3}(\mathbf{1 2}) \\
& \mathrm{R}=n-\mathrm{C}_{3} \mathrm{H}_{7}, \mathrm{X}=\mathrm{Y}=\mathrm{Z}=\mathrm{F}(\mathbf{1 3})
\end{aligned}
$$

$$
\begin{aligned}
& \mathrm{R}=n-\mathrm{C}_{5} \mathrm{H}_{11}, \mathrm{X}=\mathrm{Y}=\mathrm{H}, \mathrm{Z}=\mathrm{OCF}_{3}(20) \\
& \mathrm{R}=n-\mathrm{C}_{5} \mathrm{H}_{11}, \mathrm{X}=\mathrm{Y}=\mathrm{H}, \mathrm{Z}=\mathrm{F}(\mathbf{2 1}) \\
& \mathrm{R}=n-\mathrm{C}_{5} \mathrm{H}_{11}, \mathrm{X}=\mathrm{F}, \mathrm{Y}=\mathrm{H}, \mathrm{Z}=\mathrm{OCF}_{3}(\mathbf{2 2}) \\
& \mathrm{R}=n-\mathrm{C}_{5} \mathrm{H}_{11}, \mathrm{X}=\mathrm{Z}=\mathrm{F}, \mathrm{Y}=\mathrm{H}(\mathbf{2 3}) \\
& \mathrm{R}=n-\mathrm{C}_{5} \mathrm{H}_{11}, \mathrm{X}=\mathrm{Y}=\mathrm{F}, \mathrm{Z}=\mathrm{OCF}_{3}(24) \\
& \mathrm{R}=n-\mathrm{C}_{5} \mathrm{H}_{11}, \mathrm{X}=\mathrm{Y}=\mathrm{Z}=\mathrm{F}(\mathbf{2 5})
\end{aligned}
$$




\section{Results and Discussion}

\subsection{Synthesis of BPEBs and Analogues (1-25)}

The synthetic routes of 1-25 are outlined in Scheme 2, including the key steps of the formation of terminal and internal alkynes via Sonogashira cross-coupling reactions of aryl iodides/bromides catalyzed by palladium( 0 ) complexes in good to high yields, and a typical synthetic procedure for the formation of $\mathbf{1 2}$ is described in the Experimental Section (vide infra).

Scheme 2. Synthesis of 1,4-bis(phenylethynyl)benzene derivatives and analogues.

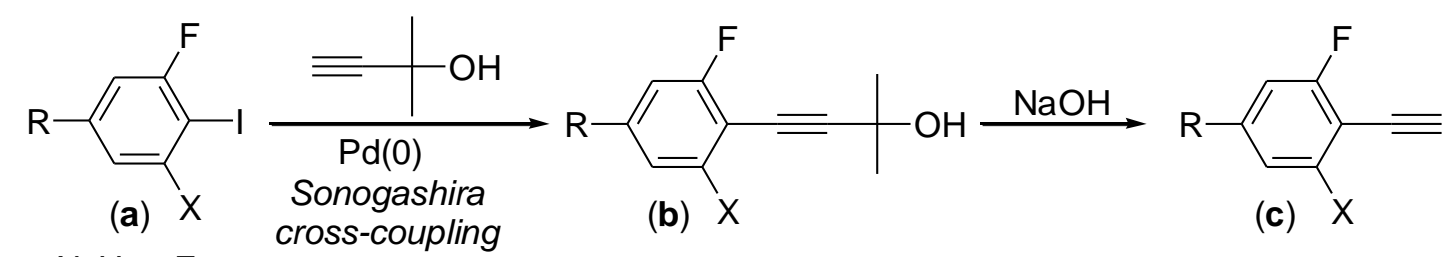

$\mathrm{X}: \mathrm{H}$ or $\mathrm{F}$<smiles>[X]c1cc(C)cc(I)c1I</smiles>

(d) $\mathrm{Y}^{\prime}$

$\mathrm{n}=1$ or 2

$X^{\prime}, Y^{\prime}: H$ or $F$

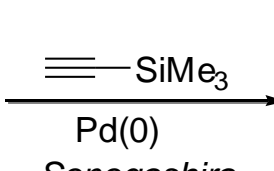

Sonogashira cross-coupling

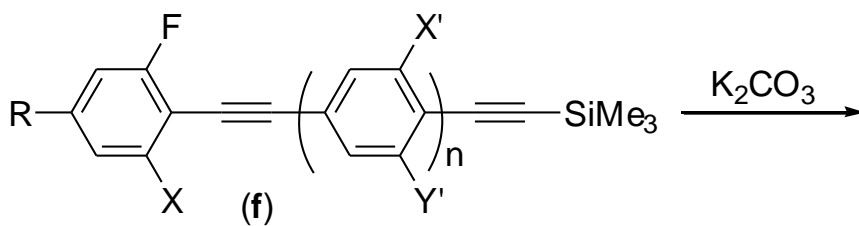

$(\mathbf{f})$

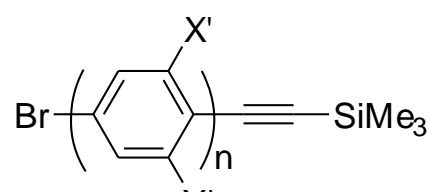

(e) $Y^{\prime}$

(c) +

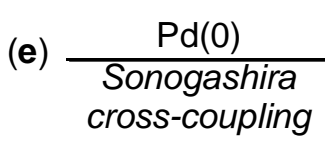

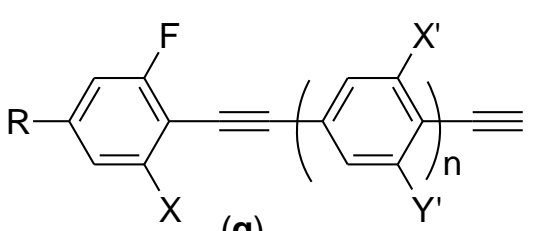

(g)

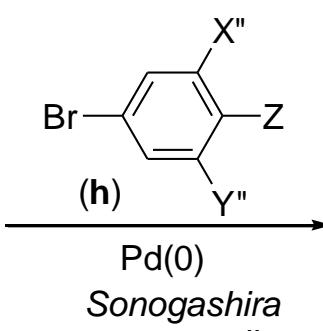

cross-coupling 1,4-bis(phenylethynyl)benzene derivatives

1 - 25

\subsection{Thermal Properties}

The thermal properties of the melting point (mp) and clearing point (cp) are critical in the practical utilization of the synthesized BPEBs as the composition of LCs, thus the mp and cp were determined by DSC (Differential Scanning Calorimetry), and their thermal data as well as enthalpic data $(\Delta H)$ are concluded in Table 1. It was found that $\mathrm{mp}$ and $\mathrm{cp}$ greatly depended upon the molecular and electronic structures of BPEBs. BPEBs 1-4 clearly show the effect of alkyl chain length on mp and cp, and when $n-\mathrm{C}_{3} \mathrm{H}_{7}$ and $\mathrm{C}_{2} \mathrm{H}_{5}$ groups are used as the end groups, BPEBs $\mathbf{1}$ and $\mathbf{2}$ give the similar mp and cp (1 vs. 2). However, when the longer alkyl chains of C4 and C5 were employed, BPEBs 3 and 4 showed similar thermal properties, but both $\mathrm{mp}$ and $\mathrm{cp}$ decreased greatly ( $1 \& 2$ vs. 3 \& 4) [23]. It can be concluded that BPEBs $\mathbf{3}$ and $\mathbf{4}$ bearing the longer alkyl chains have a wider range of nematic phase 
temperature than those of BPEBs 1 and 2, possibly due to the longer alkyl chains being more flexible than short alkyl chains. Both $\mathbf{3}$ and $\mathbf{4}$ show a nematic phase temperature range of about $140{ }^{\circ} \mathrm{C}$, and the wide nematic phase temperature are very important to make a practical LC mixture.

Table 1. Melting points and clearing points of 1,4-bis(phenylethynyl)benzene derivatives (BPEBs) and analogues by DSC.

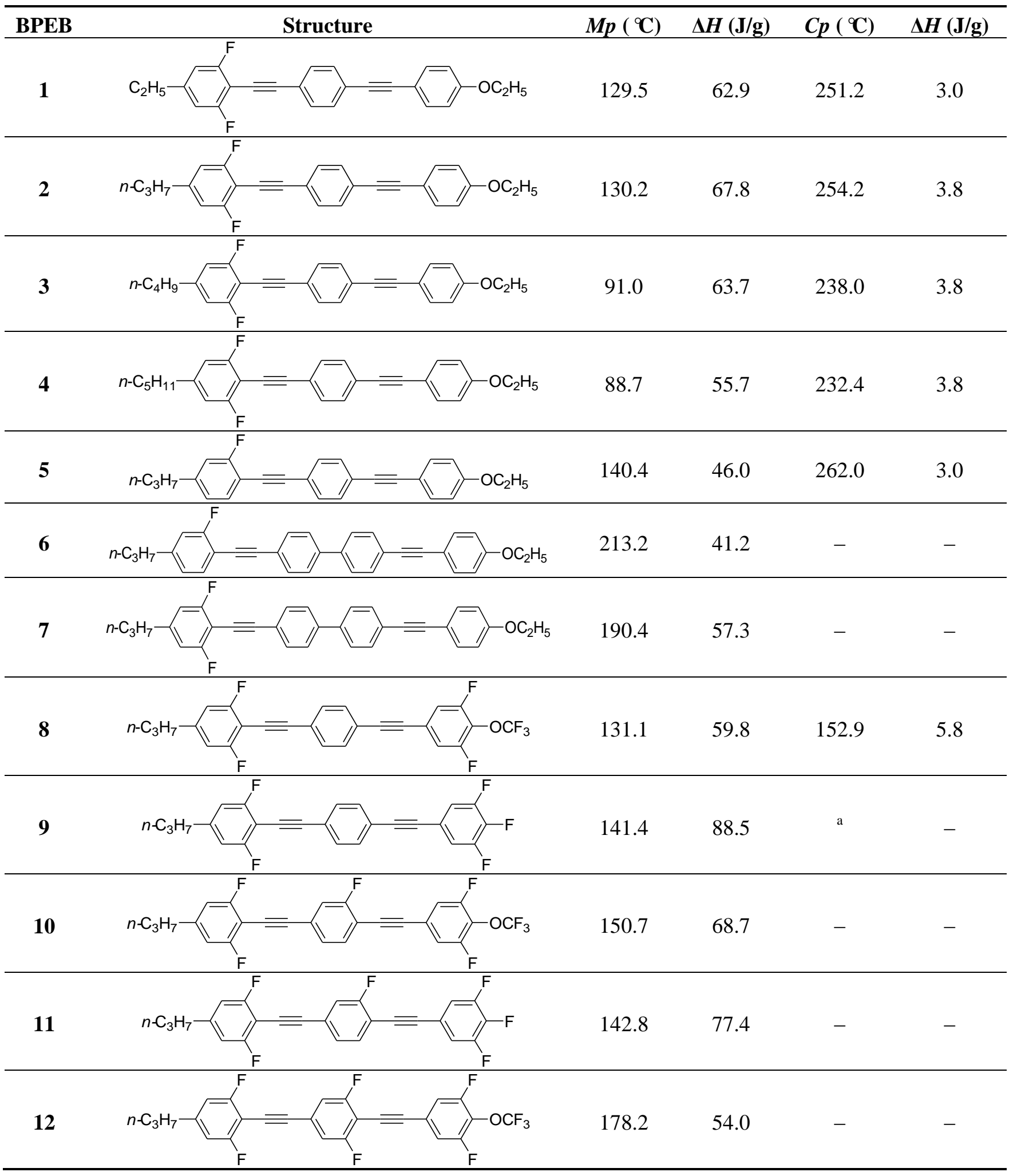


Table 1. Cont.

\begin{tabular}{|c|c|c|c|c|c|}
\hline BPEB & Structure & $M p\left({ }^{\circ} \mathbf{C}\right)$ & $\Delta \mathbf{H}(\mathbf{J} / \mathbf{g})$ & $C p\left({ }^{\circ} \mathbf{C}\right)$ & $\Delta \mathbf{H}(\mathrm{J} / \mathrm{g})$ \\
\hline 13 & & 176.4 & 66.5 & - & - \\
\hline 14 & & 142.6 & 69.3 & b & - \\
\hline 15 & & 125.2 & 73.6 & c & - \\
\hline 16 & & 147.8 & 45.7 & - & - \\
\hline 17 & & 136.2 & 55.4 & - & - \\
\hline 18 & & 178.3 & 64.6 & - & - \\
\hline 19 & & 170.8 & 55.3 & - & - \\
\hline 20 & & 131.3 & 59.7 & 144.3 & 0.81 \\
\hline 21 & & 107.7 & 62.5 & 122.3 & 0.29 \\
\hline 22 & & 131.3 & 41.2 & - & - \\
\hline 23 & & 126.0 & 51.7 & - & - \\
\hline 24 & & 171.1 & 66.8 & - & - \\
\hline 25 & & 158.7 & 66.0 & - & - \\
\hline
\end{tabular}

${ }^{a}$ The nematic phase was only observed during cooling in the temperature range 118.3 to $89.3{ }^{\circ} \mathrm{C}$, and iso- $N 118.3{ }^{\circ} \mathrm{C}(\Delta H=0.25 \mathrm{~J} / \mathrm{g}), \mathrm{N}-\mathrm{Cr} 89.3{ }^{\circ} \mathrm{C}(\Delta H=60.4 \mathrm{~J} / \mathrm{g}){ }^{\mathrm{b}}{ }^{\mathrm{b}}$ The nematic phase was only observed during cooling in the temperature range 139.6 to $121.4{ }^{\circ} \mathrm{C}$, and iso- $N 139.6{ }^{\circ} \mathrm{C}(\Delta H=0.71 \mathrm{~J} / \mathrm{g}), \mathrm{N}-\mathrm{Sm} 121.4{ }^{\circ} \mathrm{C}$ $(\Delta H=0.61 \mathrm{~J} / \mathrm{g}), \mathrm{Sm}-\mathrm{Cr} 113.8^{\circ} \mathrm{C}(\Delta H=60.27 \mathrm{~J} / \mathrm{g}){ }^{\mathrm{c}}{ }^{\mathrm{c}}$ The nematic phase was only observed during cooling in the temperature range 107.0 to $71.4{ }^{\circ} \mathrm{C}$, and iso- $N 107.0{ }^{\circ} \mathrm{C}(\Delta H=0.16 \mathrm{~J} / \mathrm{g}), \mathrm{N}-\mathrm{Cr} 71.4{ }^{\circ} \mathrm{C}(\Delta H=59.1 \mathrm{~J} / \mathrm{g})$. 
By comparison of $\mathbf{2}$ and $\mathbf{5}$, it was found that decreasing the number of fluorine atoms in the end benzene ring leads to the increase of both $\mathrm{mp}$ and $\mathrm{cp}$ in a range of about $10{ }^{\circ} \mathrm{C}$, and a similar trend of mp was also found between 4,4'-bis(phenylethynyl)biphenyls 6 and 7, which are the analogues of BPEBs 2 or 5.

The thermal properties of other BPEBs are also compared to each other, as shown in Figure 1, and two notable features of the relationship between mps and their chemical structure could be concluded as follows: (1) The introduction of the fluorine atom on the middle benzene ring shows a great effect on the change of mp. In general, one or two fluorine atom-substituted benzene result in the increase of $\mathrm{mp}$, and the introduction of the second fluorine atom leads to much more significant increase of $\mathrm{mp}$ relative to the first fluorine atom introduction (e.g., $\mathbf{8} \rightarrow \mathbf{1 0} \rightarrow \mathbf{1 2} ; \mathbf{9} \rightarrow \mathbf{1 1} \rightarrow \mathbf{1 3}$ ). (2) When the end group of $\mathrm{OCF}_{3}$ is replaced by a fluorine atom, the mps decreased (e.g., 10 vs. 11; 12 vs. 13; 14 vs. 15), and only one exception (8vs. 9) was observed.

Figure 1. Effect of side-substituted fluorine atoms and end groups on melting points.

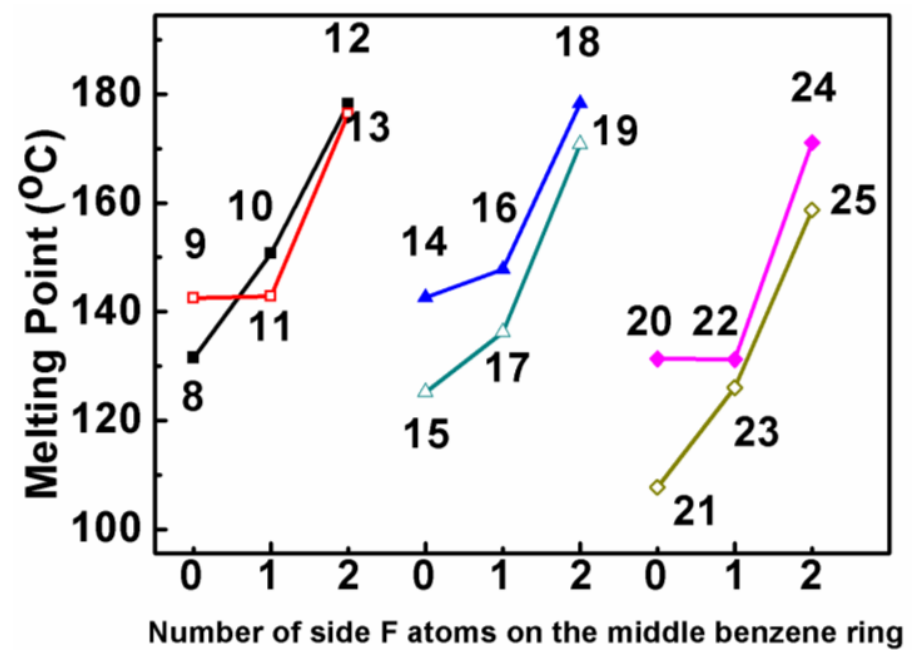

\subsection{Nematic Phase}

Among the synthesis of BPEBs and analogues, only BPEBs 1-5, 8, 20-21 (Figure 2, on heating run) and 9, 14-15 (on cooling run) show nematic phases under polarizing microscope with the structural character without side-substituted fluorine atoms bonded to the middle benzene ring, and the analogues of BPEBs 6 and 7 have no nematic phase either. It was found that the nematic phase temperature is quite different depending on the end groups and the numbers of fluorine atoms. As shown in Figure 3 and Table 1, BPEBs 1-5 with $\mathrm{OC}_{2} \mathrm{H}_{5}$ as the end group possess wide nematic phase temperatures from 121.6 to $147.0{ }^{\circ} \mathrm{C}$, and have apparently disclosed that longer alkyl chains generally result in a wider temperature of nematic phase. BPEBs $\mathbf{3}$ and $\mathbf{4}$ bearing the longer alkyl chain of $n-\mathrm{C}_{4} \mathrm{H}_{9}$ or $n-\mathrm{C}_{5} \mathrm{H}_{11}$ give the maximum nematic phase temperatures, while BPEBs 8,20 and 21 with $\mathrm{OCF}_{3}$ or $\mathrm{F}$ as the end groups show the relatively narrow nematic phase temperature range. 
Figure 2. Polarizing optical micrographs photos of BPEBs 1-5, 8, 9, 14, 15, 20 and 21.
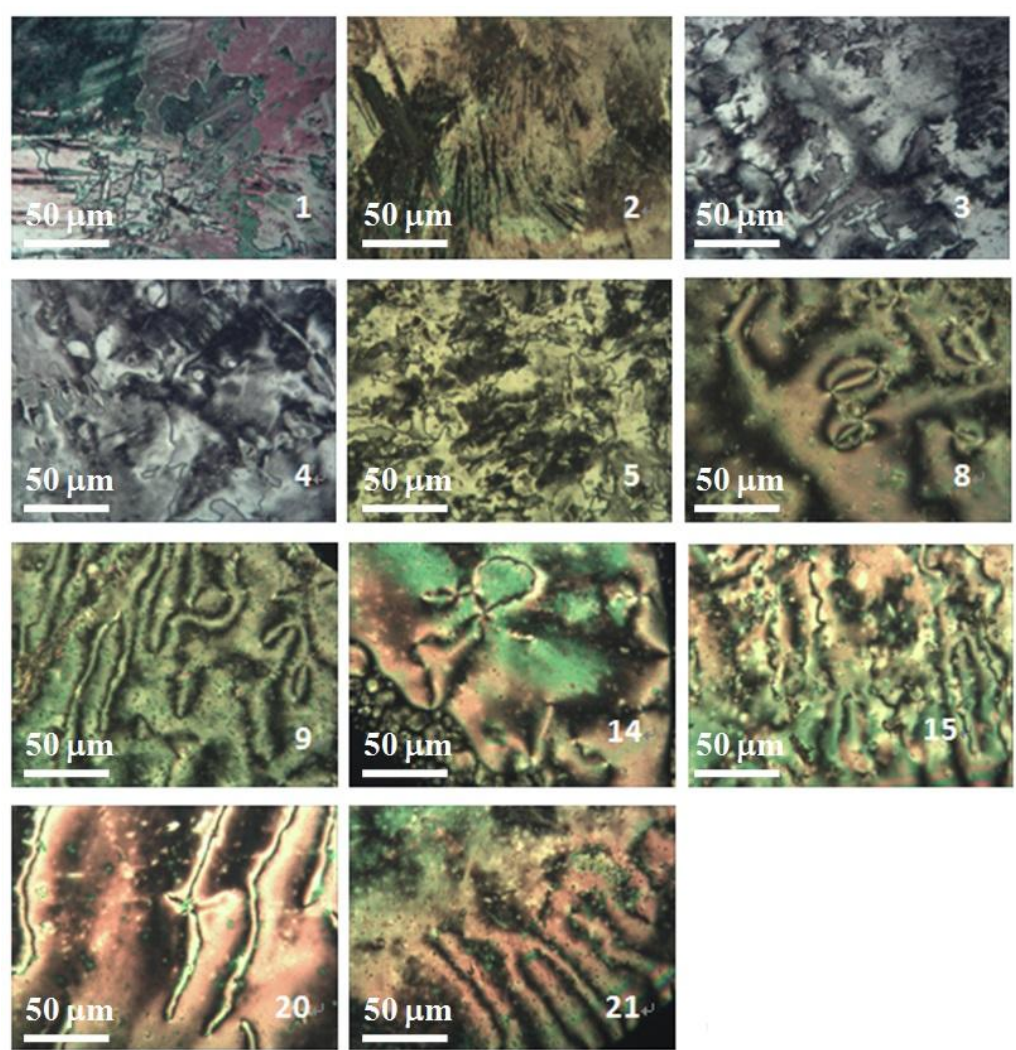

Figure 3. Nematic phase temperature range.

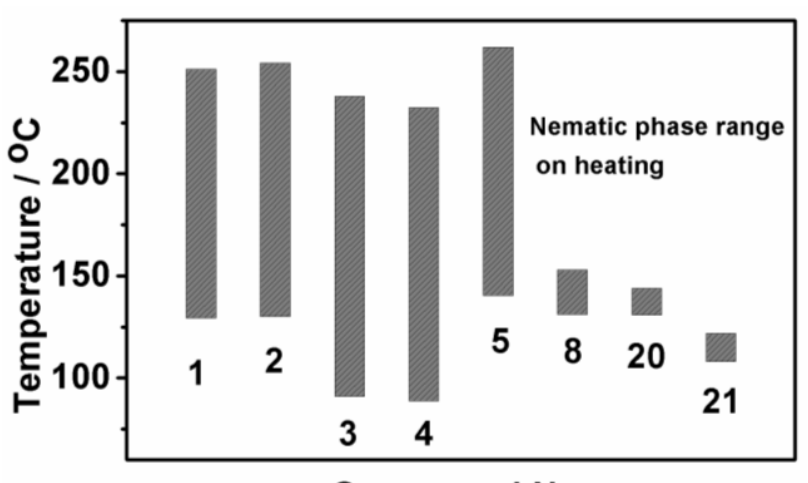

Compound No.

\subsection{Optical Anisotropy (Un)}

The optical anisotropy or birefringence $(\Delta n)$ of the BPEBs showing nematic phase in host LC was determined, and the obtained results were concluded in Table 2. As expected, a large $\pi$-conjugated structure leads to relatively high $\Delta n$ value, and therefore BPEBs 1-5 have higher $\Delta n$ values than 8-9, 14-15, and 20-21. It is reasonable to understand that much more side-substituted fluorine atoms in different benzene rings will decrease the integrity of $\pi$-conjugated structures to result in a decrease of optical anisotropy. 
Table 2. $\Delta n$ values of BPEBs.

\begin{tabular}{cccccccc}
\hline $\mathbf{B P E B}$ & $\boldsymbol{n}_{\mathrm{e}}$ & $\boldsymbol{n}_{\mathbf{0}}$ & $\boldsymbol{\Delta} \boldsymbol{n}$ & $\mathbf{B P E B}$ & $\boldsymbol{n}_{\mathrm{e}}$ & $\boldsymbol{n}_{\mathbf{0}}$ & $\boldsymbol{\Delta} \boldsymbol{n}$ \\
\hline $\mathbf{1}$ & 2.027 & 1.531 & 0.496 & $\mathbf{9}$ & 1.867 & 1.521 & 0.346 \\
$\mathbf{2}$ & 1.987 & 1.511 & 0.476 & $\mathbf{1 4}$ & 1.797 & 1.468 & 0.329 \\
$\mathbf{3}$ & 1.947 & 1.511 & 0.436 & $\mathbf{1 5}$ & 1.867 & 1.521 & 0.346 \\
$\mathbf{4}$ & 2.007 & 1.511 & 0.496 & $\mathbf{2 0}$ & 1.807 & 1.491 & 0.316 \\
$\mathbf{5}$ & 2.103 & 1.521 & 0.582 & $\mathbf{2 1}$ & 1.867 & 1.511 & 0.356 \\
$\mathbf{8}$ & 1.837 & 1.491 & 0.346 & & & & \\
\hline \multicolumn{7}{c}{ : Extraordinary refraction index; $n_{\mathrm{o}}$ : Ordinary refraction index. }
\end{tabular}

\subsection{Dielectric Anisotropy ( $\Delta \varepsilon)$}

Table 3 shows the values of dielectric anisotropy $(\Delta \varepsilon)$ of the BPEBs having nematic phase in host LC. It was found that with the number increase of side-substituted fluorine atoms, BPEBs 8-9, 14-15, and 20-21 have higher dielectric anisotropy values than BPEBs 1-5. In addition, comparison of BPEBs with the end groups of $\mathrm{OCF}_{3}(\mathbf{8}, \mathbf{1 4}, \mathbf{2 0})$ and $\mathrm{F}(\mathbf{9}, \mathbf{1 5}, \mathbf{2 1}), \mathrm{BPEBs} 8,14$ and 20 have relatively higher dielectric anisotropy values, possibly due to the stronger electronegativity of the $\mathrm{OCF}_{3}$ group relative to the fluorine atom.

Table 3. $\Delta \varepsilon$ values of BPEBs.

\begin{tabular}{cccccccc}
\hline $\mathbf{B P E B}$ & $\boldsymbol{\varepsilon}_{/ /}$ & $\boldsymbol{\varepsilon}_{\perp}$ & $\boldsymbol{\Delta} \boldsymbol{\varepsilon}$ & $\mathbf{B P E B}$ & $\boldsymbol{\varepsilon}_{/ /}$ & $\boldsymbol{\varepsilon}_{\perp}$ & $\boldsymbol{\Delta} \boldsymbol{\varepsilon}$ \\
\hline $\mathbf{1}$ & 8.9 & 3.4 & 5.5 & $\mathbf{9}$ & 26.1 & 5.3 & 20.8 \\
$\mathbf{2}$ & 8.0 & 3.0 & 5.0 & $\mathbf{1 4}$ & 29.5 & 6.0 & 23.5 \\
$\mathbf{3}$ & 7.2 & 3.0 & 4.2 & $\mathbf{1 5}$ & 26.4 & 5.4 & 21.0 \\
$\mathbf{4}$ & 8.1 & 3.0 & 5.1 & $\mathbf{2 0}$ & 26.1 & 5.2 & 20.9 \\
$\mathbf{5}$ & 7.3 & 3.2 & 4.1 & $\mathbf{2 1}$ & 25.0 & 5.0 & 20.0 \\
$\mathbf{8}$ & 29.5 & 5.5 & 24.0 & & & & \\
\hline
\end{tabular}

$\varepsilon_{/ /}$: Parallel dielectric constant; $\varepsilon_{\perp}$ : Vertical dielectric constant.

\subsection{Applications of BPEBs as Blue Phase Liquid Crystal Composition}

Because the synthesized some of BPEBs have high $\Delta n$ and acceptable $\Delta \varepsilon$ as described above, we are interested in investigation of the application of them as the compositions in blue phase liquid crystals (BPLC) to possibly increase the Kerr constant, which is key parameter for practical BPLC. After detailed screening the composition and contents, we got a LC mixture containing BPEBs 1, 3-4, 8-9, 14, 15, 21 (5 wt \% each) and other liquid crystal mixture (cp: $83.0{ }^{\circ} \mathrm{C} ; \Delta n=0.230$, and $\Delta \varepsilon=29.6$, at $25^{\circ} \mathrm{C}$ ), which shows the properties of $\Delta n=0.283$, and $\Delta \varepsilon=29.0$ at $25^{\circ} \mathrm{C}$, which is expected to have high potential applications as BPLC [24]. After adding chiral dopants (R811: $10 \mathrm{wt} \%$ and BDH1281: $7 \mathrm{wt} \%$ ), we obtained a BPLC with a blue phase temperature range of $8 \mathrm{~K}$ (from 41 to $33{ }^{\circ} \mathrm{C}$, on the second cooling run). Figure 4 shows a typical BP texture of the obtained BPLC at $36{ }^{\circ} \mathrm{C}$. 
Figure 4. The typical blue phase texture.

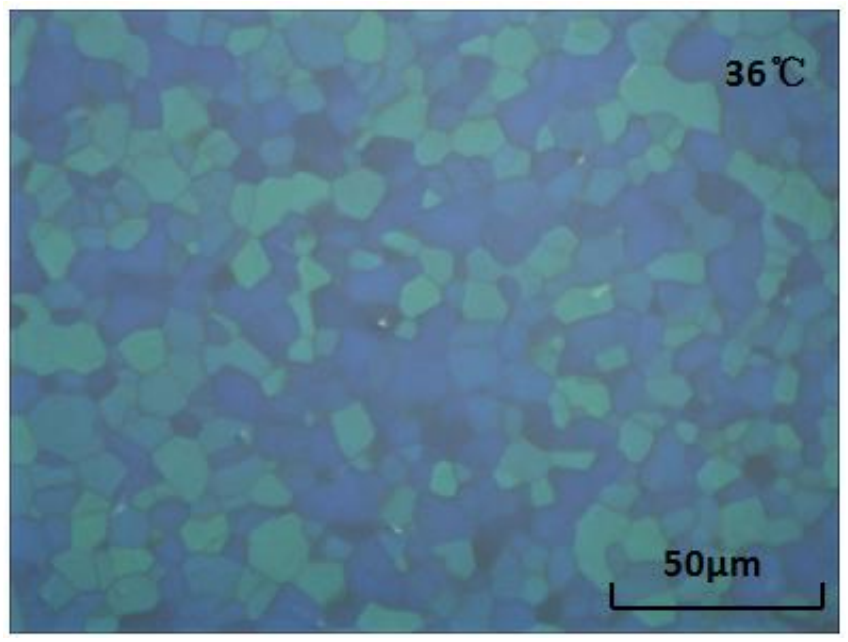

\section{Experimental Section}

\subsection{General Method}

All organic starting materials and catalysts are analytically pure and used without further purification. Nuclear magnetic resonance (NMR) spectra were recorded on a JEOL ECA-300 spectrometer (Tokyo, Japan) using $\mathrm{CDCl}_{3}$ as solvent at $298 \mathrm{~K} .{ }^{1} \mathrm{H}-\mathrm{NMR}(300 \mathrm{MHz})$ chemical shifts $(\delta)$ were referenced to internal standard TMS (for $1 \mathrm{H}, \delta=0.00 \mathrm{ppm}) .{ }^{13} \mathrm{C}-\mathrm{NMR}(75 \mathrm{MHz})$ chemical shifts were referenced to internal solvent $\mathrm{CDCl}_{3}$ (for ${ }^{13} \mathrm{C}, \delta=77.16 \mathrm{ppm}$ ). Mass spectra (MS) were obtained on a Shimadzu GCMS-QP2010S (Kyoto, Japan). Element analyses were obtained with a Flash EA 1112 Element Analyzer (Thermo Fisher Scientific, Waltham, MA, USA). Polarizing microscope LWT300LPT (CEWEI photoelectric technology Co. Ltd., Shanghai, China) equipped with a Weitu WT-3000 hot stage and a TCA 5.0 MP camera was used to observe and record the optical textures of liquid crystal samples. The calorimetric studies were performed on a TA Instruments DSC 2010 (TA Instruments, New Castle, DE, USA) with a heating temperature rate of $10{ }^{\circ} \mathrm{C} / \mathrm{min}$. A NAR-4T Abbe refractometer (ATAGO Co. Ltd., Tokyo, Japan) was used to measure optical anisotropy $(\Delta n)$, and a 3522-50 LCR Hitester (HIOKI E.E. Co., Ueda, Japan) for dielectric anisotropy $(\Delta \varepsilon)$. All the samples for measuring the $\Delta n$ and $\Delta \varepsilon$ were composed of BPEB and nematic host LC at a ratio of 5-10/95-90 (wt \%/wt \%). The nematic host LC (SLC960524) was prepared by our laboratory, which has the values of $\Delta n=0.1202(589 \mathrm{~nm})$ and $\Delta \varepsilon=3.121(1000 \mathrm{~Hz})$ at $25^{\circ} \mathrm{C}$.

3.2. A Typical Experimental Procedure for Synthesis of BPEB 12 and the Characterization Data of All the BPEBs

As shown in Scheme 2, BPEBs and analogues (1-25) were synthesized by the similar synthetic route, and their structures were characterized by ${ }^{1} \mathrm{H}-\mathrm{NMR},{ }^{13} \mathrm{C}-\mathrm{NMR}$ (for BPEBs 6 and 7, the ${ }^{13} \mathrm{C}-\mathrm{NMR}$ could not be obtained due to their very low solubility in $\mathrm{CDCl}_{3}$, DMSO- $d_{6}$ or DMF- $d_{7}$ ), and elemental analyses. In this section, the synthetic procedure of BPEB 12 was only described in details, and the characterization data of all the other BPEBs are given. 
Preparation of 2,6-difluoro-4- $n$-propylphenyl acetylene (12c) (See Scheme S1).

2-Methyl-3-butyn-2-ol (21.8 g, $0.3 \mathrm{~mol})$ was added to a mixture of 2,6-difluoro-4- $n$-propyl-1iodobenzene $(56.4 \mathrm{~g}, 0.2 \mathrm{~mol})$, tetrakis(triphenylphosphine) palladium(0) (1.0 g, $0.87 \mathrm{mmol})$, $\mathrm{CuBr}(0.5 \mathrm{~g})$ and $\mathrm{LiBr}(2.0 \mathrm{~g})$ in triethylamine $(50 \mathrm{~mL})$ with stirring at room temperature. After the mixture was heated at $60{ }^{\circ} \mathrm{C}$ for $5 \mathrm{~h}$, it was then cooled to room temperature and the saturated $\mathrm{NH}_{4} \mathrm{Cl}$ aqueous solution $(100 \mathrm{~mL})$ and ethyl acetate $(200 \mathrm{~mL})$ were added. After separation of the organic phase, the aqueous phase was extracted by ethyl acetate $(3 \times 150 \mathrm{~mL})$, and the combined organic extracts are dried by $\mathrm{K}_{2} \mathrm{CO}_{3}$ and concentrated on a rotary evaporator, the intermediate $\mathbf{1 2 b}$ was obtained by column chromatographic separation ( $40.5 \mathrm{~g}, 0.17 \mathrm{~mol}, 85.0 \%)$ for the next reaction.

Sodium hydroxide $(20.0 \mathrm{~g}, 0.5 \mathrm{~mol})$ was added to a solution of $\mathbf{1 2 b}(40.0 \mathrm{~g})$ in toluene $(150 \mathrm{~mL})$, and then the obtained mixture was heated under reflux for $5 \mathrm{~h}$. After removal of the insoluble excess of Sodium hydroxide by filtration and the solvent under reduced pressure, 12c was isolated by column chromatography as light yellow oil (17.2 g, $0.095 \mathrm{~mol}, 55.9 \%)$. Characterization data for 12c: ${ }^{1} \mathrm{H}-\mathrm{NMR}\left(300 \mathrm{MHz}, \mathrm{CDCl}_{3}\right) \delta 6.70\left(\mathrm{~d}, 2 \mathrm{H},{ }^{3} \mathrm{~J}_{\mathrm{F}-\mathrm{C}-\mathrm{C}-\mathrm{H}}=8.4 \mathrm{~Hz}\right), 6.67(\mathrm{~s}, 1 \mathrm{H}), 3.44(\mathrm{~s}, 1 \mathrm{H}), 2.52(\mathrm{t}, 2 \mathrm{H}$, $J=7.5 \mathrm{~Hz}), 1.61-1.54(\mathrm{~m}, 2 \mathrm{H}), 0.89(\mathrm{t}, 3 \mathrm{H}, J=7.5 \mathrm{~Hz}) ;{ }^{13} \mathrm{C}-\mathrm{NMR}\left(75 \mathrm{MHz}, \mathrm{CDCl}_{3}\right) \delta 163.5$ $\left(\mathrm{dd},{ }^{1} J_{\mathrm{C}-\mathrm{F}}=253.6 \mathrm{~Hz},{ }^{5} J_{\mathrm{C}-\mathrm{F}}=6.0 \mathrm{~Hz}\right), 147.1\left(\mathrm{t},{ }^{3} J_{\mathrm{C}-\mathrm{F}}=9.0 \mathrm{~Hz}\right), 111.1\left(\mathrm{dd},{ }^{2} J_{\mathrm{C}-\mathrm{F}}=17.1 \mathrm{~Hz}\right.$, $\left.{ }^{4} J_{\mathrm{C}-\mathrm{F}}=6.0 \mathrm{~Hz}\right), 98.2\left(\mathrm{t},{ }^{2} J_{\mathrm{C}-\mathrm{F}}=19.8 \mathrm{~Hz}\right), 86.5,70.9,37.8,23.7,13.4$; MS $\mathrm{m} / \mathrm{z}$ (\% rel. intensity) 180 ( $\mathrm{M}^{+}$, 44), 151 (100); Anal. calcd for $\mathrm{C}_{11} \mathrm{H}_{10} \mathrm{~F}_{2}$ : C, 73.33; H, 5.56. Found: C, 73.62; H, 5.59.

Preparation of [2,6-difluoro-4-(2',6'-difluoro-4'-n-propylphenyl)ethynyl]phenyl acetylene (12g) (See Scheme S2).

A mixture of 2-bromo-1,3-difluoro-5-iodobenzene (11.16 g, $0.035 \mathrm{~mol})$, ethynyl trimethylsilane (3.8 g, $0.039 \mathrm{~mol})$, tetrakis(triphenylphosphine) palladium(0) $(0.5 \mathrm{~g}, 0.44 \mathrm{mmol})$ and $\mathrm{CuI}(1.0 \mathrm{~g})$ in triethylamine $(40.0 \mathrm{~mL})$ was stirred in under argon at room temperature for $16 \mathrm{~h}$. After work-up as described for 12b, 12e was obtained in $85.7 \%$ (10.0 g, $0.03 \mathrm{~mol})$.

A mixture of 12e $(5.6 \mathrm{~g}), 12 \mathrm{c}(3.0 \mathrm{~g}, 0.017 \mathrm{~mol})$, tetrakis(triphenylphosphine)palladium $(0)$ $(0.2 \mathrm{~g}, 0.17 \mathrm{mmol})$ and $\mathrm{CuI}(0.4 \mathrm{~g})$ in triethylamine $(40.0 \mathrm{~mL})$ was heated with string at $60{ }^{\circ} \mathrm{C}$ for $5 \mathrm{~h}$, After work-up as described for $\mathbf{1 2 b}$, the intermediated $\mathbf{1 2 f}$ was obtained as colorless solid in $76.4 \%$ (4.8 g, $0.013 \mathrm{~mol})$. And then stirring a mixture of $\mathbf{1 2 f}(4.8 \mathrm{~g}, 0.013 \mathrm{~mol})$ and $\mathrm{K}_{2} \mathrm{CO}_{3}(0.1 \mathrm{~g}, 0.7 \mathrm{mmol})$ in methanol $(150 \mathrm{~mL})$ at room temperature for $5 \mathrm{~h}$, after work up as described for the isolation of 12c, $\mathbf{1 2 g}$ was isolated as colorless solid in $84.6 \%(3.47 \mathrm{~g}, 0.011 \mathrm{~mol})$. Characterization data for 12g: ${ }^{1} \mathrm{H}-\mathrm{NMR}\left(300 \mathrm{MHz}, \mathrm{CDCl}_{3}\right) \delta 7.11\left(\mathrm{~d}, 2 \mathrm{H},{ }^{3} J_{\mathrm{F}-\mathrm{C}-\mathrm{C}-\mathrm{H}}=7.5 \mathrm{~Hz}\right), 6.77\left(\mathrm{~d}, 2 \mathrm{H},{ }^{3} \mathrm{~J}_{\mathrm{F}-\mathrm{C}-\mathrm{C}-\mathrm{H}}=8.1 \mathrm{~Hz}\right)$, $3.59(\mathrm{~s}, 1 \mathrm{H}), 2.59(\mathrm{t}, 2 \mathrm{H}, J=7.5 \mathrm{~Hz}), 1.68-1.60(\mathrm{~m}, 2 \mathrm{H}), 0.94(\mathrm{t}, 3 \mathrm{H}, J=7.4 \mathrm{~Hz}) ;{ }^{13} \mathrm{C}-\mathrm{NMR}$ $\left(300 \mathrm{MHz}, \mathrm{CDCl}_{3}\right) \delta 163.3\left(\mathrm{dd},{ }^{1} J_{\mathrm{C}-\mathrm{F}}=255.0 \mathrm{~Hz},{ }^{5} J_{\mathrm{C}-\mathrm{F}}=6.6 \mathrm{~Hz}\right), 162.8\left(\mathrm{dd},{ }^{1} J_{\mathrm{C}-\mathrm{F}}=254.2 \mathrm{~Hz}\right.$, $\left.{ }^{5} J_{\mathrm{C}-\mathrm{F}}=5.8 \mathrm{~Hz}\right), 147.6\left(\mathrm{t},{ }^{3} J_{\mathrm{C}-\mathrm{F}}=9.1 \mathrm{~Hz}\right), 125.1\left(\mathrm{t},{ }^{3} J_{\mathrm{C}-\mathrm{F}}=11.9 \mathrm{~Hz}\right), 114.5\left(\mathrm{dd},{ }^{2} J_{\mathrm{C}-\mathrm{F}}=18.2 \mathrm{~Hz}\right.$, $\left.{ }^{4} J_{\mathrm{C}-\mathrm{F}}=6.8 . \mathrm{Hz}\right), 111.4\left(\mathrm{dd},{ }^{2} J_{\mathrm{C}-\mathrm{F}}=17.3 \mathrm{~Hz},{ }^{4} J_{\mathrm{C}-\mathrm{F}}=6.3 . \mathrm{Hz}\right), 102.1\left(\mathrm{t},{ }^{2} J_{\mathrm{C}-\mathrm{F}}=19.6 \mathrm{~Hz}\right), 98.4$ $\left(\mathrm{t},{ }^{2} J_{\mathrm{C}-\mathrm{F}}=19.6 \mathrm{~Hz}\right), 88.8,88.9,80.7,70.5,38.0,23.8,13.6 ; \mathrm{MS} \mathrm{m} / \mathrm{z}$ (\% rel. intensity) $316\left(\mathrm{M}^{+}, 56\right)$; 287 (100); Anal. calcd for $\mathrm{C}_{19} \mathrm{H}_{12} \mathrm{~F}_{4}$ : C, 72.15; H, 3.80. Found: C, 72.52; H, 3.98.

Preparation of 1-[(2',6'-difluoro-4'-n-propylphenyl)ethynyl]-4-[(3",5"-difluoro-4"-trifluoro-methoxyphenyl)ethynyl]-3,5-difluorobenzene (BPEB 12) (See Scheme S3).

A solution of 4-bromo-2,6-difluoro(trifluoromethoxy)benzene $(2.2 \mathrm{~g}, 8.04 \mathrm{mmol})$ in toluene $(10.0 \mathrm{~mL})$ was added into a solution of $\mathbf{1 2} \mathbf{g}(1.69 \mathrm{~g}, 5.36 \mathrm{mmol})$, tetrakis(triphenylphosphine)palladium(0) $(0.1 \mathrm{~g}$, $0.09 \mathrm{mmol})$, and $\mathrm{CuI}(0.2 \mathrm{~g})$ in triethylamine $(20.0 \mathrm{~mL})$ under argon at $60{ }^{\circ} \mathrm{C}$, and then the obtained 
mixture was stirred at $60{ }^{\circ} \mathrm{C}$ for $5 \mathrm{~h}$. After work-up as described for 12c, the desired BPEB 12 was isolated as colorless solid in 79.1\% (2.17 g, $4.24 \mathrm{mmol})$. Characterization data for BPEB 12: ${ }^{1} \mathrm{H}-\mathrm{NMR}$ $\left(300 \mathrm{MHz}, \mathrm{CDCl}_{3}\right) \delta 7.24\left(\mathrm{~d}, 2 \mathrm{H},{ }^{3} J_{\mathrm{F}-\mathrm{C}-\mathrm{C}-\mathrm{H}}=7.5\right), 7.15\left(\mathrm{~d}, 2 \mathrm{H},{ }^{3} J_{\mathrm{F}-\mathrm{C}-\mathrm{C}-\mathrm{H}}=7.5 \mathrm{~Hz}\right), 6.78(\mathrm{~d}, 2 \mathrm{H}$, $\left.{ }^{3} J_{\mathrm{F}-\mathrm{C}-\mathrm{C}-\mathrm{H}}=8.4 \mathrm{~Hz}\right), 2.60(\mathrm{t}, 2 \mathrm{H}, J=7.5 \mathrm{~Hz}), 1.69-1.61(\mathrm{~m}, 2 \mathrm{H}), 0.95(\mathrm{t}, 3 \mathrm{H}, J=7.4 \mathrm{~Hz}) ;{ }^{13} \mathrm{C}-\mathrm{NMR}$ $\left(75 \mathrm{MHz}, \mathrm{CDCl}_{3}\right) \delta 162.9\left(\mathrm{dd},{ }^{1} J_{\mathrm{C}-\mathrm{F}}=254.3 \mathrm{~Hz},{ }^{5} J_{\mathrm{C}-\mathrm{F}}=5.7 \mathrm{~Hz}\right), 162.6\left(\mathrm{dd},{ }^{1} J_{\mathrm{C}-\mathrm{F}}=254.8 \mathrm{~Hz}\right.$, $\left.{ }^{5} J_{\mathrm{C}-\mathrm{F}}=6.0 \mathrm{~Hz}\right), 155.9\left(\mathrm{dd},{ }^{1} J_{\mathrm{C}-\mathrm{F}}=253.9 \mathrm{~Hz},{ }^{5} J_{\mathrm{C}-\mathrm{F}}=3.6 \mathrm{~Hz}\right), 147.7\left(\mathrm{t},{ }^{3} J_{\mathrm{C}-\mathrm{F}}=9.0 \mathrm{~Hz}\right), 126.3$ $\left(\mathrm{t},{ }^{3} J_{\mathrm{C}-\mathrm{F}}=15.6 \mathrm{~Hz}\right), 125.6\left(\mathrm{t},{ }^{3} J_{\mathrm{C}-\mathrm{F}}=12.1 \mathrm{~Hz}\right), 123.0\left(\mathrm{t},{ }^{3} J_{\mathrm{C}-\mathrm{F}}=10.6 \mathrm{~Hz}\right), 120.5\left(\mathrm{q},{ }^{1} J_{\mathrm{C}-\mathrm{F}}=260.3 \mathrm{~Hz}\right)$, $116.0\left(\mathrm{dd},{ }^{2} J_{\mathrm{C}-\mathrm{F}}=16.2 \mathrm{~Hz},{ }^{4} J_{\mathrm{C}-\mathrm{F}}=5.0 \mathrm{~Hz}\right), 114.6\left(\mathrm{dd},{ }^{2} J_{\mathrm{C}-\mathrm{F}}=18.2 \mathrm{~Hz},{ }^{4} J_{\mathrm{C}-\mathrm{F}}=7.0 \mathrm{~Hz}\right), 111.4$ $\left(\mathrm{dd},{ }^{2} J_{\mathrm{C}-\mathrm{F}}=17.2 \mathrm{~Hz},{ }^{4} J_{\mathrm{C}-\mathrm{F}}=5.7 \mathrm{~Hz}\right), 102.0\left(\mathrm{t},{ }^{2} J_{\mathrm{C}-\mathrm{F}}=18.7 \mathrm{~Hz}\right), 98.5\left(\mathrm{t},{ }^{2} J_{\mathrm{C}-\mathrm{F}}=19.7 \mathrm{~Hz}\right), 96.8,95.5$, 81.1, 79.2, 38.0, 23.8, 13.6; Anal. calcd for $\mathrm{C}_{26} \mathrm{H}_{13} \mathrm{~F}_{9} \mathrm{O}$ : C, 60.93; H, 2.54. Found: C, 61.33; H, 2.50 .

Characterization data for BPEB 1: ${ }^{1} \mathrm{H}-\mathrm{NMR}\left(300 \mathrm{MHz}, \mathrm{CDCl}_{3}\right) \delta 7.56-7.45(\mathrm{~m}, 6 \mathrm{H}), 6.87(\mathrm{~d}, 2 \mathrm{H}$, $J=8.8 \mathrm{~Hz}), 6.78(\mathrm{~d}, 2 \mathrm{H}, J=8.2 \mathrm{~Hz}), 4.04(\mathrm{q}, 2 \mathrm{H}, J=7.0 \mathrm{~Hz}), 2.65(\mathrm{q}, 2 \mathrm{H}, J=7.6 \mathrm{~Hz}), 1.42(\mathrm{t}, 3 \mathrm{H}$, $J=7.0 \mathrm{~Hz}), 1.24(\mathrm{t}, 3 \mathrm{H}, J=7.6 \mathrm{~Hz}) ;{ }^{13} \mathrm{C}-\mathrm{NMR}\left(75 \mathrm{MHz}, \mathrm{CDCl}_{3}\right) \delta 162.8(\mathrm{dd}, J=253.1 \mathrm{~Hz}$, $J=6.0 \mathrm{~Hz}), 159.3,147.9(\mathrm{t}, J=8.9 \mathrm{~Hz}), 133.2,131.7,131.4,124.1,122.2,115.0,114.7,110.7$ $(\mathrm{dd}, J=16.9 \mathrm{~Hz}, J=6.3 \mathrm{~Hz}), 99.4(\mathrm{t}, J=19.9 \mathrm{~Hz}), 98.1,91.8,87.9,78.9,63.6,28.9,14.9,14.8$; Anal. calcd for $\mathrm{C}_{26} \mathrm{H}_{20} \mathrm{~F}_{2} \mathrm{O}$ : C, 80.83; H, 5.18. Found: C, 81.03; H, 4.90.

Characterization data for BPEB 2: ${ }^{1} \mathrm{H}-\mathrm{NMR}\left(300 \mathrm{MHz}, \mathrm{CDCl}_{3}\right) \delta 7.56-7.45(\mathrm{~m}, 6 \mathrm{H}), 6.87(\mathrm{~d}, 2 \mathrm{H}$, $J=8.7 \mathrm{~Hz}), 6.76(\mathrm{~d}, 2 \mathrm{H}, J=8.0 \mathrm{~Hz}), 4.04(\mathrm{q}, 2 \mathrm{H}, J=7.0 \mathrm{~Hz}), 2.58(\mathrm{t}, 2 \mathrm{H}, J=7.4 \mathrm{~Hz}), 1.70-1.58$ $(\mathrm{m}, 2 \mathrm{H}), 1.42(\mathrm{t}, 3 \mathrm{H}, J=7.0 \mathrm{~Hz}), 0.95(\mathrm{t}, 3 \mathrm{H}, J=7.3 \mathrm{~Hz}) ;{ }^{13} \mathrm{C}-\mathrm{NMR}\left(75 \mathrm{MHz}, \mathrm{CDCl}_{3}\right) \delta 162.8$ $(\mathrm{dd}, J=253.0 \mathrm{~Hz}, J=6.0 \mathrm{~Hz}), 159.3,146.4(\mathrm{t}, J=9.0 \mathrm{~Hz}), 133.2,131.7,131.4,124.1,122.2,115.0$, 114.7, $111.3(\mathrm{dd}, J=16.6 \mathrm{~Hz}, J=6.0 \mathrm{~Hz}), 99.4(\mathrm{t}, J=20.1 \mathrm{~Hz}), 98.1,91.8,87.9,78.3,63.6,38.0$, 23.9, 14.8, 13.7; Anal. calcd for $\mathrm{C}_{27} \mathrm{H}_{22} \mathrm{~F}_{2} \mathrm{O}$ : C, 81.00; H, 5.50. Found: C, 81.43; H, 5.74.

Characterization data for BPEB 3: ${ }^{1} \mathrm{H}-\mathrm{NMR}\left(300 \mathrm{MHz}, \mathrm{CDCl}_{3}\right) \delta 7.56-7.45(\mathrm{~m}, 6 \mathrm{H}), 6.87(\mathrm{~d}, 2 \mathrm{H}$, $J=8.7 \mathrm{~Hz}), 6.76(\mathrm{~d}, 2 \mathrm{H}, J=8.1 \mathrm{~Hz}), 4.04(\mathrm{q}, 2 \mathrm{H}, J=7.0 \mathrm{~Hz}), 2.60(\mathrm{t}, 2 \mathrm{H}, J=7.5 \mathrm{~Hz}), 1.61-1.54$ $(\mathrm{m}, 2 \mathrm{H}), 1.45-1.30(\mathrm{~m}, 5 \mathrm{H}), 0.94(\mathrm{t}, 3 \mathrm{H}, J=7.3 \mathrm{~Hz}) ;{ }^{13} \mathrm{C}-\mathrm{NMR}\left(75 \mathrm{MHz}, \mathrm{CDCl}_{3}\right) \delta 162.8$ $(\mathrm{dd}, J=253.0 \mathrm{~Hz}, J=6.1 \mathrm{~Hz}), 159.3,146.7(\mathrm{t}, J=9.0 \mathrm{~Hz}), 133.2,131.7,131.4,124.1,122.2,115.0$, 114.7, $111.2(\mathrm{dd}, J=16.8 \mathrm{~Hz}, J=6.0 \mathrm{~Hz}), 99.4(\mathrm{t}, J=20.0 \mathrm{~Hz}), 98.1,91.8,87.9,78.3,63.6,35.7$, 32.8, 22.2, 14.8, 13.9; Anal. calcd for $\mathrm{C}_{28} \mathrm{H}_{24} \mathrm{~F}_{2} \mathrm{O}$ : C, 81.16; H, 5.80. Found: C, 81.53; H, 5.84.

Characterization data for BPEB 4: ${ }^{1} \mathrm{H}-\mathrm{NMR}\left(300 \mathrm{MHz}, \mathrm{CDCl}_{3}\right) \delta 7.55-7.45(\mathrm{~m}, 6 \mathrm{H}), 6.87(\mathrm{~d}, 2 \mathrm{H}$, $J=8.7 \mathrm{~Hz}), 6.76(\mathrm{~d}, 2 \mathrm{H}, J=8.1 \mathrm{~Hz}), 4.04(\mathrm{q}, 2 \mathrm{H}, J=7.0 \mathrm{~Hz}), 2.60(\mathrm{t}, 2 \mathrm{H}, J=7.5 \mathrm{~Hz}), 1.66-1.57(\mathrm{~m}, 2 \mathrm{H})$, $1.43(\mathrm{t}, 3 \mathrm{H}, J=7.0 \mathrm{~Hz}), 1.36-1.25(\mathrm{~m}, 4 \mathrm{H}), 0.91(\mathrm{t}, 3 \mathrm{H}, J=6.9 \mathrm{~Hz}) ;{ }^{13} \mathrm{C}-\mathrm{NMR}\left(75 \mathrm{MHz}, \mathrm{CDCl}_{3}\right)$ $\delta 162.8(\mathrm{dd}, J=253.2 \mathrm{~Hz}, J=6.1 \mathrm{~Hz}), 159.3,146.7(\mathrm{t}, J=9.0 \mathrm{~Hz}), 133.2,131.7,131.4,124.1,122.2$, 115.0, 114.6, $111.2(\mathrm{dd}, J=17.0 \mathrm{~Hz}, J=6.1 \mathrm{~Hz}), 99.4(\mathrm{t}, J=19.5 \mathrm{~Hz}), 98.1,91.8,87.9,78.3,63.6$, 35.9, 31.4, 30.4, 22.5, 14.8, 14.1; Anal. calcd for $\mathrm{C}_{29} \mathrm{H}_{26} \mathrm{~F}_{2} \mathrm{O}: \mathrm{C}, 81.31$; H, 6.07. Found: C, 81.26; H, 6.34. Characterization data for BPEB 5: ${ }^{1} \mathrm{H}-\mathrm{NMR}\left(300 \mathrm{MHz}, \mathrm{CDCl}_{3}\right) \delta 7.54-7.39(\mathrm{~m}, 7 \mathrm{H}), 6.94(\mathrm{~d}, 2 \mathrm{H}$, $J=8.4 \mathrm{~Hz}), 6.87(\mathrm{~d}, 2 \mathrm{H}, J=8.3 \mathrm{~Hz}), 4.04(\mathrm{q}, 2 \mathrm{H}, J=6.7 \mathrm{~Hz}), 2.60(\mathrm{t}, 3 \mathrm{H}, J=7.3 \mathrm{~Hz}), 1.72-1.59$ $(\mathrm{m}, 2 \mathrm{H}), 1.43(\mathrm{t}, 3 \mathrm{H}, J=6.8 \mathrm{~Hz}), 0.96(\mathrm{t}, 3 \mathrm{H}, J=7.2 \mathrm{~Hz}) ;{ }^{13} \mathrm{C}-\mathrm{NMR}\left(75 \mathrm{MHz}, \mathrm{CDCl}_{3}\right) \delta 162.7$ (d, $J=251.6 \mathrm{~Hz}), 159.3,146.1$ (d, $J=7.2 \mathrm{~Hz}), 133.2,133.1,131.6,131.4,124.3,123.8,122.6,115.5$ 
(d, $J=20.3 \mathrm{~Hz}), 114.9$ (d, $J=27.2 \mathrm{~Hz}), 114.7,108.9$ (d, $J=16.0 \mathrm{~Hz}), 93.6,91.6,88.0,84.8,63.6$, 37.9, 24.1, 14.9, 13.8; Anal. calcd for $\mathrm{C}_{27} \mathrm{H}_{23}$ FO: C, 84.82; H, 6.02. Found: C, 84.88; H, 6.13.

Characterization data for BPEB 6: ${ }^{1} \mathrm{H}-\mathrm{NMR}\left(300 \mathrm{MHz}, \mathrm{CDCl}_{3}\right) \delta$ 7.64-7.53 (m, 8H), 7.49-7.45 $(\mathrm{m}, 2 \mathrm{H}), 7.41(\mathrm{~d}, 1 \mathrm{H}, J=7.5 \mathrm{~Hz}), 6.96-6.92(\mathrm{~m}, 2 \mathrm{H}), 6.88(\mathrm{~d}, 2 \mathrm{H}, J=8.7 \mathrm{~Hz}), 4.06(\mathrm{q}, 2 \mathrm{H}, J=7.0 \mathrm{~Hz})$, $2.61(\mathrm{t}, 2 \mathrm{H}, J=7.3 \mathrm{~Hz}), 1.72-1.60(\mathrm{~m}, 2 \mathrm{H}), 1.43(\mathrm{t}, 3 \mathrm{H}, J=7.0 \mathrm{~Hz}), 0.95(\mathrm{t}, 3 \mathrm{H}, J=7.3 \mathrm{~Hz})$; Anal. calcd for $\mathrm{C}_{33} \mathrm{H}_{27} \mathrm{FO}$ : C, 86.46; H, 5.90. Found: C, 86.98; H, 5.93.

Characterization data for BPEB 7: ${ }^{1} \mathrm{H}-\mathrm{NMR}\left(300 \mathrm{MHz}, \mathrm{CDCl}_{3}\right) \delta 7.66-7.55(\mathrm{~m}, 8 \mathrm{H}), 7.48(\mathrm{~d}, 2 \mathrm{H}$, $J=8.8 \mathrm{~Hz}), 6.88(\mathrm{~d}, 2 \mathrm{H}, J=8.8 \mathrm{~Hz}), 6.77(\mathrm{~d}, 2 \mathrm{H}, J=8.1 \mathrm{~Hz}), 4.06(\mathrm{q}, 2 \mathrm{H}, J=7.0 \mathrm{~Hz}), 2.59(\mathrm{t}, 2 \mathrm{H}$, $J=7.4 \mathrm{~Hz}), 1.71-1.59(\mathrm{~m}, 2 \mathrm{H}), 1.43(\mathrm{t}, 3 \mathrm{H}, J=7.0 \mathrm{~Hz}), 0.95(\mathrm{t}, 3 \mathrm{H}, J=7.4 \mathrm{~Hz})$; Anal. calcd for $\mathrm{C}_{33} \mathrm{H}_{26} \mathrm{~F}_{2} \mathrm{O}: \mathrm{C}, 83.19$; H, 5.46. Found: C, 83.26; H, 5.72.

Characterization data for BPEB 8: ${ }^{1} \mathrm{H}-\mathrm{NMR}\left(300 \mathrm{MHz}, \mathrm{CDCl}_{3}\right) \delta 7.58-7.48(\mathrm{~m}, 4 \mathrm{H}), 7.17(\mathrm{~d}, 2 \mathrm{H}$, $J=7.8 \mathrm{~Hz}), 6.77(\mathrm{~d}, 2 \mathrm{H}, J=8.1 \mathrm{~Hz}), 2.59(\mathrm{t}, 2 \mathrm{H}, J=7.4 \mathrm{~Hz}), 1.71-1.59(\mathrm{~m}, 2 \mathrm{H}), 0.95(\mathrm{t}, 3 \mathrm{H}$, $J=7.3 \mathrm{~Hz}) ;{ }^{13} \mathrm{C}-\mathrm{NMR}\left(75 \mathrm{MHz}, \mathrm{CDCl}_{3}\right) \delta 162.8(\mathrm{dd}, J=253.3 \mathrm{~Hz}, J=5.8 \mathrm{~Hz}), 155.8(\mathrm{dd}, J=255.8 \mathrm{~Hz}$, $J=3.6 \mathrm{~Hz}), 146.8(\mathrm{t}, J=8.9 \mathrm{~Hz}), 131.8,131.7,125.6(\mathrm{t}, J=17.2 \mathrm{~Hz}), 123.8(\mathrm{t}, J=9.1 \mathrm{~Hz}), 122.1$, $120.6(\mathrm{q}, J=259.6 \mathrm{~Hz}), 115.8(\mathrm{~d}, J=17.5 \mathrm{~Hz}, J=6.2 \mathrm{~Hz}), 111.3(\mathrm{dd}, J=16.9 \mathrm{~Hz}, J=6.1 \mathrm{~Hz}), 99.2$ (t, $J=19.9 \mathrm{~Hz}$ ), 97.6, 92.0, 87.9, 79.1, 38.0, 23.8, 13.6; Anal. calcd for $\mathrm{C}_{26} \mathrm{H}_{15} \mathrm{~F}_{7} \mathrm{O}: \mathrm{C}, 65.55 ; \mathrm{H}, 3.15$. Found: C, 64.98; H, 3.13.

Characterization data for BPEB 9: ${ }^{1} \mathrm{H}-\mathrm{NMR}\left(300 \mathrm{MHz}, \mathrm{CDCl}_{3}\right) \delta$ 7.57-7.47 (m, 4H), 7.16-7.11 $(\mathrm{m}, 2 \mathrm{H}), 6.76(\mathrm{~d}, 2 \mathrm{H}, J=8.1 \mathrm{~Hz}), 2.59$ (t, 2H, $J=7.4 \mathrm{~Hz}), 1.71-1.58(\mathrm{~m}, 2 \mathrm{H}), 0.95(\mathrm{t}, 3 \mathrm{H}, J=7.3 \mathrm{~Hz})$; ${ }^{13} \mathrm{C}-\mathrm{NMR}\left(75 \mathrm{MHz}, \mathrm{CDCl}_{3}\right) \delta 162.8(\mathrm{dd}, J=253.1 \mathrm{~Hz}, J=5.8 \mathrm{~Hz}), 151.1$ (ddd, $J=251.1 \mathrm{~Hz}$, $J=10.4 \mathrm{~Hz}, J=4.9 \mathrm{~Hz}), 146.7(\mathrm{t}, J=9.0 \mathrm{~Hz}), 140.5(\mathrm{dt}, J=255.7 \mathrm{~Hz}, J=15.3 \mathrm{~Hz}), 131.8,131.7$, 123.5, 122.4, 119.09 (td), 116.0 (ddd, $J=15.2 \mathrm{~Hz}, J=7.3 \mathrm{~Hz}), 111.3(\mathrm{dd}, J=17.0 \mathrm{~Hz}, J=5.9 \mathrm{~Hz}$ ), $99.2(\mathrm{t}, J=20.0 \mathrm{~Hz}), 97.7,90.6,88.2,78.9$, 38.0, 23.9, 13.6; Anal. calcd for $\mathrm{C}_{25} \mathrm{H}_{15} \mathrm{~F}_{5}$ : C, 73.17; H, 3.66. Found: C, 73.02; H, 3.51.

Characterization data for BPEB 10: ${ }^{1} \mathrm{H}-\mathrm{NMR}\left(300 \mathrm{MHz}, \mathrm{CDCl}_{3}\right) \delta 7.47(\mathrm{t}, 1 \mathrm{H}, \mathrm{J}=7.6 \mathrm{~Hz}), 7.35-7.30$ $(\mathrm{m}, 2 \mathrm{H}), 7.20(\mathrm{~d}, 2 \mathrm{H}, J=7.8 \mathrm{~Hz}), 6.77(\mathrm{~d}, 2 \mathrm{H}, J=8.2 \mathrm{~Hz}), 2.59(\mathrm{t}, 2 \mathrm{H}, J=7.4 \mathrm{~Hz}), 1.71-1.59(\mathrm{~m}, 2 \mathrm{H})$, $0.95(\mathrm{t}, 3 \mathrm{H}, J=7.3 \mathrm{~Hz}) ;{ }^{13} \mathrm{C}-\mathrm{NMR}\left(75 \mathrm{MHz}, \mathrm{CDCl}_{3}\right) \delta 162.8(\mathrm{dd}, J=253.9 \mathrm{~Hz}, J=6.0 \mathrm{~Hz}), 162.3$ $(\mathrm{d}, J=253.5 \mathrm{~Hz}), 155.8(\mathrm{dd}, J=255.4 \mathrm{~Hz}, J=3.8 \mathrm{~Hz}), 147.2(\mathrm{t}, J=9.1 \mathrm{~Hz}), 133.3,127.6(\mathrm{~d}, J=3.2 \mathrm{~Hz})$, $126.1(\mathrm{t}, J=15.8 \mathrm{~Hz}), 125.6(\mathrm{~d}, J=9.3 \mathrm{~Hz}), 123.3(\mathrm{t}, J=11.0 \mathrm{~Hz}), 120.5(\mathrm{q}, J=261.0 \mathrm{~Hz}), 118.7$ $(\mathrm{d}, J=22.7 \mathrm{~Hz}), 115.9(\mathrm{dd}, J=15.5 \mathrm{~Hz}, J=4.3 \mathrm{~Hz}), 111.4(\mathrm{dd}, J=17.5 \mathrm{~Hz}, J=5.6 \mathrm{~Hz}), 111.1,98.8$ (t, $J=19.6 \mathrm{~Hz}$ ), 96.4, 92.6, 85.5, 80.0, 40.0, 23.8, 13.6; Anal. calcd for $\mathrm{C}_{26} \mathrm{H}_{14} \mathrm{~F}_{8} \mathrm{O}: \mathrm{C}, 63.16 ; \mathrm{H}, 2.83$. Found: C, 63.03; H, 2.56.

Characterization data for BPEB 11: ${ }^{1} \mathrm{H}-\mathrm{NMR}\left(300 \mathrm{MHz}, \mathrm{CDCl}_{3}\right) \delta 7.45(\mathrm{t}, 1 \mathrm{H}, J=7.4 \mathrm{~Hz}), 7.36-7.26$ $(\mathrm{m}, 2 \mathrm{H}), 7.21-7.14(\mathrm{~m}, 2 \mathrm{H}), 6.77(\mathrm{~d}, 2 \mathrm{H}, J=8.2 \mathrm{~Hz}), 2.59(\mathrm{t}, 2 \mathrm{H}, J=7.3 \mathrm{~Hz}), 1.71-1.58(\mathrm{~m}, 2 \mathrm{H})$, $0.95(\mathrm{t}, 3 \mathrm{H}, J=7.3 \mathrm{~Hz}) ;{ }^{13} \mathrm{C}-\mathrm{NMR}\left(75 \mathrm{MHz}, \mathrm{CDCl}_{3}\right) \delta 162.8(\mathrm{dd}, J=253.6 \mathrm{~Hz}, J=5.6 \mathrm{~Hz}), 162.3$ $(\mathrm{d}, J=253.2 \mathrm{~Hz}), 151.1(\mathrm{ddd}, J=251.0 \mathrm{~Hz}, J=10.5 \mathrm{~Hz}, J=4.8 \mathrm{~Hz}), 147.2(\mathrm{t}, J=8.9 \mathrm{~Hz}), 140.6$ $(\mathrm{dt}, J=250.7 \mathrm{~Hz}, J=10.9 \mathrm{~Hz}), 133.3,127.6(\mathrm{~d}, J=3.3 \mathrm{~Hz}), 125.3(\mathrm{~d}, J=9.3 \mathrm{~Hz}), 118.6(\mathrm{dd}, J=12.4 \mathrm{~Hz}$, 
$J=6.9 \mathrm{~Hz}), 116.1(\mathrm{dd}, J=15.5 \mathrm{~Hz}, J=7.4 \mathrm{~Hz}), 111.4,111.4(\mathrm{dd}, J=17.8 \mathrm{~Hz}, J=6.3 \mathrm{~Hz}), 98.9$ (t, $J=19.4 \mathrm{~Hz}), 96.5(\mathrm{~d}, J=3.2 \mathrm{~Hz}), 92.9,84.1,79.9,38.0,23.8,13.6$; Anal. calcd for $\mathrm{C}_{25} \mathrm{H}_{14} \mathrm{~F}_{6}$ : C, 70.09; H, 3.27. Found: C, 70.33; H, 3.46.

Characterization data for BPEB 13: ${ }^{1} \mathrm{H}-\mathrm{NMR}\left(300 \mathrm{MHz}, \mathrm{CDCl}_{3}\right) \delta 7.22-7.12(\mathrm{~m}, 4 \mathrm{H}), 6.78(\mathrm{~d}, 2 \mathrm{H}$, $J=8.2 \mathrm{~Hz}), 2.60(\mathrm{t}, 2 \mathrm{H}, J=7.4 \mathrm{~Hz}), 1.71-1.59(\mathrm{~m}, 2 \mathrm{H}), 0.95(\mathrm{t}, 3 \mathrm{H}, J=7.3 \mathrm{~Hz}) ;{ }^{13} \mathrm{C}-\mathrm{NMR}(75 \mathrm{MHz}$, $\left.\mathrm{CDCl}_{3}\right) \delta 162.8(\mathrm{dd}, J=254.0 \mathrm{~Hz}, J=5.4 \mathrm{~Hz}), 162.6(\mathrm{dd}, J=255.1 \mathrm{~Hz}, J=6.4 \mathrm{~Hz}), 151.2(\mathrm{ddd}$, $J=252.3 \mathrm{~Hz}, J=10.7 \mathrm{~Hz}, J=4.4 \mathrm{~Hz}), 147.6(\mathrm{t}, J=6.4 \mathrm{~Hz}), 140.9(\mathrm{dt}, J=256.0 \mathrm{~Hz}, J=15.4 \mathrm{~Hz})$, $125.3(\mathrm{t}, J=12.2 \mathrm{~Hz}), 118.4,116.2(\mathrm{dd}, J=15.5 \mathrm{~Hz}, J=7.6 \mathrm{~Hz}), 114.5(\mathrm{dd}, J=17.7 \mathrm{~Hz}, J=7.1 \mathrm{~Hz})$, $111.4(\mathrm{dd}, J=16.4 \mathrm{~Hz}, J=5.1 \mathrm{~Hz}), 102.3$ (t, $J=21.5 \mathrm{~Hz}), 98.6(\mathrm{t}, J=20.2 \mathrm{~Hz}), 97.3,95.6,80.9,77.8$, 38.0, 23.7, 13.5; Anal. calcd for $\mathrm{C}_{25} \mathrm{H}_{13} \mathrm{~F}_{7}$ : C, 67.26; H, 2.91. Found: C, 69.52; H, 2.66.

Characterization data for BPEB 14: ${ }^{1} \mathrm{H}-\mathrm{NMR}\left(300 \mathrm{MHz}, \mathrm{CDCl}_{3}\right) \delta 7.57-7.48(\mathrm{~m}, 4 \mathrm{H}), 7.17(\mathrm{~d}, 2 \mathrm{H}$, $J=7.9 \mathrm{~Hz}), 6.77(\mathrm{~d}, 2 \mathrm{H}, J=8.1 \mathrm{~Hz}), 2.61(\mathrm{t}, 2 \mathrm{H}, J=7.5 \mathrm{~Hz}), 1.65-1.55(\mathrm{~m}, 2 \mathrm{H}), 1.42-1.30(\mathrm{~m}, 2 \mathrm{H})$, $0.94(\mathrm{t}, 3 \mathrm{H}, J=7.3 \mathrm{~Hz}) ;{ }^{13} \mathrm{C}-\mathrm{NMR}\left(75 \mathrm{MHz}, \mathrm{CDCl}_{3}\right) \delta 162.8(\mathrm{dd}, J=253.4 \mathrm{~Hz}, J=6.2 \mathrm{~Hz}), 155.8$ $(\mathrm{dd}, J=255.3 \mathrm{~Hz}, J=3.7 \mathrm{~Hz}), 147.0(\mathrm{t}, J=9.1 \mathrm{~Hz}), 131.8,131.7,125.8(\mathrm{t}, J=16.3 \mathrm{~Hz}), 123.8$ $(\mathrm{t}, J=8.8 \mathrm{~Hz}), 122.1,120.4(\mathrm{q}, J=258.9 \mathrm{~Hz}), 115.8(\mathrm{dd}, J=17.5 \mathrm{~Hz}, J=6.2 \mathrm{~Hz}), 111.3(\mathrm{dd}, J=16.7$ $\mathrm{Hz}, J=5.7 \mathrm{~Hz}), 99.2(\mathrm{t}, J=20.1 \mathrm{~Hz}), 97.6,92.0,87.9,79.0,35.7,32.8,22.3$, 13.9; Anal. calcd for $\mathrm{C}_{27} \mathrm{H}_{17} \mathrm{~F}_{7} \mathrm{O}: \mathrm{C}, 66.12 ; \mathrm{H}, 3.47$. Found: $\mathrm{C}, 66.01 ; \mathrm{H}, 3.36$.

Characterization data for BPEB 15: ${ }^{1} \mathrm{H}-\mathrm{NMR}\left(300 \mathrm{MHz}, \mathrm{CDCl}_{3}\right) \delta 7.56-7.46(\mathrm{~m}, 4 \mathrm{H}), 7.13(\mathrm{t}, 2 \mathrm{H}$, $J=6.9 \mathrm{~Hz}), 6.76(\mathrm{~d}, 2 \mathrm{H}, J=8.1 \mathrm{~Hz}), 2.60(\mathrm{t}, 2 \mathrm{H}, J=7.4 \mathrm{~Hz}), 1.64-1.54(\mathrm{~m}, 2 \mathrm{H}), 1.42-1.30(\mathrm{~m}, 2 \mathrm{H})$, $0.94(\mathrm{t}, 3 \mathrm{H}, J=7.3 \mathrm{~Hz}) ;{ }^{13} \mathrm{C}-\mathrm{NMR}\left(75 \mathrm{MHz}, \mathrm{CDCl}_{3}\right) \delta 162.8(\mathrm{dd}, J=253.4 \mathrm{~Hz}, J=6.0 \mathrm{~Hz})$, $151.1(\mathrm{ddd}, J=250.6 \mathrm{~Hz}, J=10.1 \mathrm{~Hz}, J=4.2 \mathrm{~Hz}), 146.9(\mathrm{t}, J=9.0 \mathrm{~Hz}), 140.4(\mathrm{dt}, J=254.7 \mathrm{~Hz}$, $J=14.9 \mathrm{~Hz}), 131.8,131.7,123.5,122.4,119.1(\mathrm{td}), 116.0(\mathrm{ddd}, J=15.4 \mathrm{~Hz}, J=7.3 \mathrm{~Hz}, J=4.8 \mathrm{~Hz})$, $111.2(\mathrm{dd}, J=17.1 \mathrm{~Hz}, J=5.9 \mathrm{~Hz}), 99.2$ (t, $J=19.8 \mathrm{~Hz}), 97.7,90.6,88.2,78.9,35.7,32.8,22.3,13.9$; Anal. calcd for $\mathrm{C}_{26} \mathrm{H}_{17} \mathrm{~F}_{5}$ : C, 73.58; H, 4.01. Found: C, 73.51; H, 3.96 .

Characterization data for BPEB 16: ${ }^{1} \mathrm{H}-\mathrm{NMR}\left(300 \mathrm{MHz}, \mathrm{CDCl}_{3}\right) \delta 7.47(\mathrm{t}, 1 \mathrm{H}, J=7.5 \mathrm{~Hz}), 7.39-7.29$ $(\mathrm{m}, 2 \mathrm{H}), 7.20(\mathrm{~d}, 2 \mathrm{H}, J=7.7 \mathrm{~Hz}), 6.77(\mathrm{~d}, 2 \mathrm{H}, J=8.2 \mathrm{~Hz}), 2.61(\mathrm{t}, 2 \mathrm{H}, J=7.6 \mathrm{~Hz}), 1.65-1.55(\mathrm{~m}, 2 \mathrm{H})$, $1.42-1.30(\mathrm{~m}, 2 \mathrm{H}), 0.94(\mathrm{t}, 3 \mathrm{H}, J=7.3 \mathrm{~Hz}) ;{ }^{13} \mathrm{C}-\mathrm{NMR}\left(75 \mathrm{MHz}, \mathrm{CDCl}_{3}\right) \delta 162.8(\mathrm{dd}, J=253.8 \mathrm{~Hz}$, $J=5.8 \mathrm{~Hz}), 162.3(\mathrm{~d}, J=253.7 \mathrm{~Hz}), 155.8(\mathrm{dd}, J=255.5 \mathrm{~Hz}, J=3.7 \mathrm{~Hz}), 147.5(\mathrm{t}, J=8.9 \mathrm{~Hz}), 133.3$, $127.6(\mathrm{~d}, J=3.4 \mathrm{~Hz}), 126.1(\mathrm{t}, J=15.8 \mathrm{~Hz}), 125.6(\mathrm{~d}, J=9.4 \mathrm{~Hz}), 123.3(\mathrm{t}, J=11.2 \mathrm{~Hz}), 120.5$ $(\mathrm{q}, J=261.0 \mathrm{~Hz}), 118.7(\mathrm{~d}, J=22.8 \mathrm{~Hz}), 115.9(\mathrm{dd}, J=16.2 \mathrm{~Hz}, J=6.4 \mathrm{~Hz}), 111.3(\mathrm{dd}, J=16.1 \mathrm{~Hz}$, $\mathrm{J}=5.8 \mathrm{~Hz}), 111.1,98.8(\mathrm{t}, J=19.5 \mathrm{~Hz}), 96.4,92.5,85.5,80.0,35.7,32.8,22.3$, 13.9; Anal. calcd for $\mathrm{C}_{27} \mathrm{H}_{16} \mathrm{~F}_{8} \mathrm{O}: \mathrm{C}, 63.78 ; \mathrm{H}, 3.15$. Found: $\mathrm{C}, 63.61 ; \mathrm{H}, 3.26$.

Characterization data for BPEB 17: ${ }^{1} \mathrm{H}-\mathrm{NMR}\left(300 \mathrm{MHz}, \mathrm{CDCl}_{3}\right) \delta 7.44(\mathrm{t}, 1 \mathrm{H}, J=7.4 \mathrm{~Hz}), 7.33-7.27$ $(\mathrm{m}, 2 \mathrm{H}), 7.16(\mathrm{t}, 2 \mathrm{H}, J=6.6 \mathrm{~Hz}), 6.76(\mathrm{~d}, 2 \mathrm{H}, J=8.2 \mathrm{~Hz}), 2.60(\mathrm{t}, 2 \mathrm{H}, J=7.5 \mathrm{~Hz}), 1.64-1.54(\mathrm{~m}, 2 \mathrm{H})$, $1.42-1.29(\mathrm{~m}, 2 \mathrm{H}), 0.94(\mathrm{t}, 3 \mathrm{H}, J=7.3 \mathrm{~Hz}) ;{ }^{13} \mathrm{C}-\mathrm{NMR}\left(75 \mathrm{MHz}, \mathrm{CDCl}_{3}\right) \delta 162.8(\mathrm{dd}, J=253.7 \mathrm{~Hz}$, $J=5.8 \mathrm{~Hz}), 162.3(\mathrm{~d}, J=253.4 \mathrm{~Hz}), 151.1(\mathrm{ddd}, J=250.9 \mathrm{~Hz}, J=10.4 \mathrm{~Hz}, J=4.3 \mathrm{~Hz}), 147.4$ (t, $J=9.0 \mathrm{~Hz}), 140.7(\mathrm{dt}, J=255.1 \mathrm{~Hz}, J=14.7 \mathrm{~Hz}), 133.3,127.6(\mathrm{~d}, J=3.3 \mathrm{~Hz}), 125.3(\mathrm{~d}, J=9.5 \mathrm{~Hz})$, $118.6(\mathrm{dd}, J=14.2 \mathrm{~Hz}, J=9.1 \mathrm{~Hz}), 116.1(\mathrm{dd}, J=15.4 \mathrm{~Hz}, J=7.3 \mathrm{~Hz}), 111.5,111.3(\mathrm{dd}, J=17.0 \mathrm{~Hz}$, 
$\mathrm{J}=5.9 \mathrm{~Hz}), 98.8(\mathrm{t}, J=19.6 \mathrm{~Hz}), 96.5(\mathrm{~d}, J=3.1 \mathrm{~Hz}), 92.9,84.1,79.9,35.7,32.8,22.3,13.9$; Anal. calcd for $\mathrm{C}_{26} \mathrm{H}_{16} \mathrm{~F}_{6}$ : C, 70.59; H, 3.62. Found: C, 70.68; H, 3.36.

Characterization data for BPEB 18: ${ }^{1} \mathrm{H}-\mathrm{NMR}\left(300 \mathrm{MHz}, \mathrm{CDCl}_{3}\right) \delta 7.23(\mathrm{~d}, 2 \mathrm{H}, J=7.7 \mathrm{~Hz}), 7.14$ $(\mathrm{d}, 2 \mathrm{H}, J=7.3 \mathrm{~Hz}), 6.78(\mathrm{~d}, 2 \mathrm{H}, J=8.2 \mathrm{~Hz}), 2.62(\mathrm{t}, 2 \mathrm{H}, J=7.6 \mathrm{~Hz}), 1.65-1.55(\mathrm{~m}, 2 \mathrm{H}), 1.41-1.30$ $(\mathrm{m}, 2 \mathrm{H}), 0.94(\mathrm{t}, 3 \mathrm{H}, J=7.3 \mathrm{~Hz}) ;{ }^{13} \mathrm{C}-\mathrm{NMR}\left(75 \mathrm{MHz}, \mathrm{CDCl}_{3}\right) \delta 162.9(\mathrm{dd}, J=254.1 \mathrm{~Hz}, J=5.8 \mathrm{~Hz})$, $162.7(\mathrm{dd}, J=255.4 \mathrm{~Hz}, J=6.2 \mathrm{~Hz}), 155.9(\mathrm{dd}, J=255.8 \mathrm{~Hz}, J=3.6 \mathrm{~Hz}), 147.9$ (t, $J=9.0 \mathrm{~Hz}), 126.3$ $(\mathrm{t}, J=15.8 \mathrm{~Hz}), 125.6(\mathrm{t}, J=12.0 \mathrm{~Hz}), 123.0(\mathrm{t}, J=10.1 \mathrm{~Hz}), 120.5(\mathrm{q}, J=260.8 \mathrm{~Hz}), 116.0(\mathrm{dd}$, $J=17.0 \mathrm{~Hz}, J=6.6 \mathrm{~Hz}), 114.6(\mathrm{dd}, J=17.8 \mathrm{~Hz}, J=7.9 \mathrm{~Hz}), 111.3(\mathrm{dd}, J=17.0 \mathrm{~Hz}, J=5.6 \mathrm{~Hz})$, $102.0(\mathrm{t}, J=18.5 \mathrm{~Hz}), 98.2(\mathrm{t}, J=19.9 \mathrm{~Hz}), 96.9,95.5,81.1,79.2,35.7,32.7,22.2$, 13.8; Anal. calcd for $\mathrm{C}_{27} \mathrm{H}_{15} \mathrm{~F}_{9} \mathrm{O}$ : C, 61.60; H, 2.85. Found: C, 60.99; H, 2.64.

Characterization data for BPEB 19: ${ }^{1} \mathrm{H}-\mathrm{NMR}\left(300 \mathrm{MHz}, \mathrm{CDCl}_{3}\right) \delta 7.20(\mathrm{t}, 2 \mathrm{H}, J=6.7 \mathrm{~Hz}), 7.12$ $(\mathrm{d}, 2 \mathrm{H}, J=7.1 \mathrm{~Hz}), 6.77(\mathrm{~d}, 2 \mathrm{H}, J=8.2 \mathrm{~Hz}), 2.62(\mathrm{t}, 2 \mathrm{H}, J=7.5 \mathrm{~Hz}), 1.65-1.55(\mathrm{~m}, 2 \mathrm{H}), 1.42-1.30$ $(\mathrm{m}, 2 \mathrm{H}), 0.94(\mathrm{t}, 3 \mathrm{H}, J=7.3 \mathrm{~Hz}) ;{ }^{13} \mathrm{C}-\mathrm{NMR}\left(75 \mathrm{MHz}, \mathrm{CDCl}_{3}\right) \delta 162.8(\mathrm{dd}, J=254.1 \mathrm{~Hz}, J=5.7 \mathrm{~Hz})$, $162.6(\mathrm{dd}, J=254.7 \mathrm{~Hz}, J=6.2 \mathrm{~Hz}), 151.1(\mathrm{ddd}, J=251.3 \mathrm{~Hz}, J=10.4 \mathrm{~Hz}, J=4.4 \mathrm{~Hz}), 147.9$ $(\mathrm{t}, J=9.2 \mathrm{~Hz}), 140.9(\mathrm{dt}, J=256.0 \mathrm{~Hz}, J=15.6 \mathrm{~Hz}), 125.2(\mathrm{t}, J=12.0 \mathrm{~Hz}), 118.4(\mathrm{td}), 116.3$ (ddd, $J=15.4 \mathrm{~Hz}, J=7.3 \mathrm{~Hz}), 114.6(\mathrm{dd}, J=18.2 \mathrm{~Hz}, J=7.6 \mathrm{~Hz}), 111.4(\mathrm{dd}, J=17.2 \mathrm{~Hz}, J=5.7 \mathrm{~Hz})$, $102.2(\mathrm{t}, J=19.8 \mathrm{~Hz}), 98.4(\mathrm{t}, J=20.1 \mathrm{~Hz}), 97.3,95.6,80.9$, 77.8, 35.7, 32.8, 22.3, 13.9; Anal. calcd for $\mathrm{C}_{26} \mathrm{H}_{15} \mathrm{~F}_{7}$ : C, 67.83; H, 3.26. Found: C, 67.77; H, 3.44.

Characterization data for BPEB 20: ${ }^{1} \mathrm{H}-\mathrm{NMR}\left(300 \mathrm{MHz}, \mathrm{CDCl}_{3}\right) \delta 7.57-7.48(\mathrm{~m}, 4 \mathrm{H}), 7.16(\mathrm{~d}, 2 \mathrm{H}$, $J=7.7 \mathrm{~Hz}), 6.77(\mathrm{~d}, 2 \mathrm{H}, J=8.2 \mathrm{~Hz}), 2.60(\mathrm{t}, 2 \mathrm{H}, J=7.5 \mathrm{~Hz}), 1.65-1.56(\mathrm{~m}, 2 \mathrm{H}), 1.38-1.28(\mathrm{~m}, 4 \mathrm{H})$, $0.91(\mathrm{t}, 3 \mathrm{H}, J=6.7 \mathrm{~Hz}) ;{ }^{13} \mathrm{C}-\mathrm{NMR}\left(75 \mathrm{MHz}, \mathrm{CDCl}_{3}\right) \delta 162.8(\mathrm{dd}, J=253.4 \mathrm{~Hz}, J=6.0 \mathrm{~Hz}), 155.8$ $(\mathrm{dd}, J=255.4 \mathrm{~Hz}, J=3.7 \mathrm{~Hz}), 147.0(\mathrm{t}, J=9.1 \mathrm{~Hz}), 131.8,131.7,125.8(\mathrm{t}, J=16.5 \mathrm{~Hz}), 123.8$ $(\mathrm{t}, J=8.7 \mathrm{~Hz}), 122.2,120.6(\mathrm{q}, J=259.6 \mathrm{~Hz}), 115.8(\mathrm{dd}, J=17.6 \mathrm{~Hz}, J=6.4 \mathrm{~Hz}), 111.2(\mathrm{dd}, J=17.1 \mathrm{~Hz}$, $J=6.1 \mathrm{~Hz}), 99.1(\mathrm{t}, J=19.9 \mathrm{~Hz}), 97.6,92.0,87.9,79.1,35.9,31.3,30.3,22.5,14.0$; Anal. calcd for $\mathrm{C}_{28} \mathrm{H}_{19} \mathrm{~F}_{7} \mathrm{O}: \mathrm{C}, 66.67 ; \mathrm{H}, 3.77$. Found: C, 66.35; H, 3.46.

Characterization data for BPEB 21: ${ }^{1} \mathrm{H}-\mathrm{NMR}\left(300 \mathrm{MHz}, \mathrm{CDCl}_{3}\right) \delta$ 7.56-7.46 (m, 4H), 7.16-7.11 $(\mathrm{m}, 2 \mathrm{H}), 6.76(\mathrm{~d}, 2 \mathrm{H}, J=8.2 \mathrm{~Hz}), 2.60(\mathrm{t}, 2 \mathrm{H}, J=7.6 \mathrm{~Hz}), 1.66-1.56(\mathrm{~m}, 2 \mathrm{H}), 1.38-1.29(\mathrm{~m}, 4 \mathrm{H}), 0.91$ $(\mathrm{t}, 3 \mathrm{H}, J=6.6 \mathrm{~Hz}) ;{ }^{13} \mathrm{C}-\mathrm{NMR}\left(75 \mathrm{MHz}, \mathrm{CDCl}_{3}\right) \delta 162.8(\mathrm{dd}, J=253.2 \mathrm{~Hz}, J=6.0 \mathrm{~Hz}), 151.1$ (ddd, $J=250.7 \mathrm{~Hz}, J=10.3 \mathrm{~Hz}, J=4.5 \mathrm{~Hz}), 147.0(\mathrm{t}, J=8.9 \mathrm{~Hz}), 140.5(\mathrm{dt}, J=256.0 \mathrm{~Hz}, J=15.6 \mathrm{~Hz})$, 131.8, 131.7, 123.5, 122.4, $119.1(\mathrm{td}), 116.0(\mathrm{ddd}, J=15.2 \mathrm{~Hz}, J=7.2 \mathrm{~Hz}, J=4.3 \mathrm{~Hz}), 111.2(\mathrm{dd}$, $J=16.6 \mathrm{~Hz}, J=5.6 \mathrm{~Hz}), 99.2(\mathrm{t}, J=19.7 \mathrm{~Hz}), 97.7,90.6,88.2,78.9,35.9,31.3,30.4,22.5,14.0$; Anal. calcd for $\mathrm{C}_{27} \mathrm{H}_{19} \mathrm{~F}_{5}$ : C, 73.97; H, 4.34. Found: C, 73.49; H, 4.44.

Characterization data for BPEB 22: ${ }^{1} \mathrm{H}-\mathrm{NMR}\left(300 \mathrm{MHz}, \mathrm{CDCl}_{3}\right) \delta 7.46(\mathrm{t}, 1 \mathrm{H}, J=7.5 \mathrm{~Hz}), 7.34-7.29$ $(\mathrm{m}, 2 \mathrm{H}), 7.19(\mathrm{~d}, 2 \mathrm{H}, J=7.1 \mathrm{~Hz}), 6.77(\mathrm{~d}, 2 \mathrm{H}, J=8.2 \mathrm{~Hz}), 2.60(\mathrm{t}, 2 \mathrm{H}, J=7.5 \mathrm{~Hz}), 1.61(\mathrm{~m}, 2 \mathrm{H})$, $1.36-1.30(\mathrm{~m}, 4 \mathrm{H}), 0.91(\mathrm{t}, 3 \mathrm{H}, J=6.6 \mathrm{~Hz}) ;{ }^{13} \mathrm{C}-\mathrm{NMR}\left(75 \mathrm{MHz}, \mathrm{CDCl}_{3}\right) \delta 162.8(\mathrm{dd}, J=253.9 \mathrm{~Hz}$, $J=5.8 \mathrm{~Hz}), 162.3(\mathrm{~d}, J=253.6 \mathrm{~Hz}), 155.8(\mathrm{dd}, J=255.5 \mathrm{~Hz}, J=3.5 \mathrm{~Hz}), 147.5(\mathrm{t}, J=9.0 \mathrm{~Hz}), 133.3$, $127.6(\mathrm{~d}, J=3.4 \mathrm{~Hz}), 126.1(\mathrm{t}, J=15.8 \mathrm{~Hz}), 125.6(\mathrm{~d}, J=9.5 \mathrm{~Hz}), 123.3(\mathrm{t}, J=10.9 \mathrm{~Hz}), 120.5$ $(\mathrm{q}, J=261.0 \mathrm{~Hz}), 118.7(\mathrm{~d}, J=22.7 \mathrm{~Hz}), 115.9(\mathrm{dd}, J=16.0 \mathrm{~Hz}, J=6.5 \mathrm{~Hz}), 111.3(\mathrm{dd}, J=14.6 \mathrm{~Hz}$, 
$J=6.1 \mathrm{~Hz}), 111.1,98.8(\mathrm{t}, J=19.9 \mathrm{~Hz}), 96.4,92.6,85.5,80.0,36.0,31.3,30.3,22.5,14.0$; Anal. calcd for $\mathrm{C}_{28} \mathrm{H}_{18} \mathrm{~F}_{8} \mathrm{O}$ : C, 64.37; H, 3.45. Found: C, 63.89; H, 3.26.

Characterization data for BPEB 23: ${ }^{1} \mathrm{H}-\mathrm{NMR}\left(300 \mathrm{MHz}, \mathrm{CDCl}_{3}\right) \delta 7.44(\mathrm{t}, 1 \mathrm{H}, J=7.6 \mathrm{~Hz}), 7.32-7.27$ $(\mathrm{m}, 2 \mathrm{H}), 7.16(\mathrm{~d}, 2 \mathrm{H}, J=6.8 \mathrm{~Hz}), 6.77(\mathrm{~d}, 2 \mathrm{H}, J=8.1 \mathrm{~Hz}), 2.60(\mathrm{t}, 2 \mathrm{H}, \mathrm{J}=7.9 \mathrm{~Hz}), 1.61(\mathrm{~m}, 2 \mathrm{H})$, $1.36-1.29(\mathrm{~m}, 4 \mathrm{H}), 0.91(\mathrm{t}, 3 \mathrm{H}, J=6.6 \mathrm{~Hz}) ;{ }^{13} \mathrm{C}-\mathrm{NMR}\left(75 \mathrm{MHz}, \mathrm{CDCl}_{3}\right) \delta 162.8(\mathrm{dd}, J=253.8 \mathrm{~Hz}$, $J=5.8 \mathrm{~Hz}), 162.3(\mathrm{~d}, J=253.4 \mathrm{~Hz}), 151.1(\mathrm{ddd}, J=251.1 \mathrm{~Hz}, J=10.4 \mathrm{~Hz}, J=4.4 \mathrm{~Hz}), 147.4$ $(\mathrm{t}, J=9.1 \mathrm{~Hz}), 140.7(\mathrm{dt}, J=256.0 \mathrm{~Hz}, J=15.5 \mathrm{~Hz}), 133.2,127.6(\mathrm{~d}, J=3.2 \mathrm{~Hz}), 125.3(\mathrm{~d}, J=9.5 \mathrm{~Hz})$, $118.6(\mathrm{dd}, J=15.6 \mathrm{~Hz}, J=6.9 \mathrm{~Hz}), 116.1(\mathrm{dd}, J=15.3 \mathrm{~Hz}, J=7.3 \mathrm{~Hz}), 111.5,111.3(\mathrm{dd}, J=17.1 \mathrm{~Hz}$, $J=6.9 \mathrm{~Hz}), 98.9(\mathrm{t}, J=19.9 \mathrm{~Hz}), 96.5,92.9,84.1,79.9,36.0,31.4,30.3,22.5$, 14.0; Anal. calcd for $\mathrm{C}_{27} \mathrm{H}_{18} \mathrm{~F}_{6}$ : C, 71.05; H, 3.95. Found: C, 71.17; H, 4.11.

Characterization data for BPEB 24: ${ }^{1} \mathrm{H}-\mathrm{NMR}\left(300 \mathrm{MHz}, \mathrm{CDCl}_{3}\right) \delta 7.23(\mathrm{~d}, 2 \mathrm{H}, J=7.7 \mathrm{~Hz}), 7.14$ $(\mathrm{d}, 2 \mathrm{H}, J=7.2 \mathrm{~Hz}), 6.78(\mathrm{~d}, 2 \mathrm{H}, J=8.2 \mathrm{~Hz}), 2.61(\mathrm{t}, 2 \mathrm{H}, J=7.5 \mathrm{~Hz}), 1.61(\mathrm{~m}, 2 \mathrm{H}), 1.38-1.27(\mathrm{~m}, 4 \mathrm{H})$, $0.90(\mathrm{t}, 3 \mathrm{H}, J=6.9 \mathrm{~Hz}) ;{ }^{13} \mathrm{C}-\mathrm{NMR}\left(75 \mathrm{MHz}, \mathrm{CDCl}_{3}\right) \delta 162.9(\mathrm{dd}, J=254.7 \mathrm{~Hz}, J=6.4 \mathrm{~Hz}), 162.6$ $(\mathrm{dd}, J=254.8 \mathrm{~Hz}, J=6.1 \mathrm{~Hz}), 155.8(\mathrm{dd}, J=255.7 \mathrm{~Hz}, J=3.6 \mathrm{~Hz}), 148.0(\mathrm{t}, J=9.4 \mathrm{~Hz}), 126.3$ $(\mathrm{t}, J=15.8 \mathrm{~Hz}), 125.5(\mathrm{t}, J=12.6 \mathrm{~Hz}), 123.0(\mathrm{t}, J=10.6 \mathrm{~Hz}), 120.5(\mathrm{q}, J=260.4 \mathrm{~Hz}), 116.0$ $(\mathrm{dd}, J=17.5 \mathrm{~Hz}, J=6.2 \mathrm{~Hz}), 114.6(\mathrm{dd}, J=18.1 \mathrm{~Hz}, J=7.3 \mathrm{~Hz}), 111.4(\mathrm{dd}, J=17.2 \mathrm{~Hz}, J=5.5 \mathrm{~Hz})$, $102.0(\mathrm{t}, J=17.6 \mathrm{~Hz}), 98.4(\mathrm{t}, J=19.9 \mathrm{~Hz}), 96.9,95.5,81.1,79.2,36.0,31.3,30.3,22.5,14.0$; Anal. calcd for $\mathrm{C}_{28} \mathrm{H}_{17} \mathrm{~F}_{9} \mathrm{O}: \mathrm{C}, 62.22 ; \mathrm{H}, 3.15$. Found: $\mathrm{C}, 62.18 ; \mathrm{H}, 3.12$.

Characterization data for BPEB 25: ${ }^{1} \mathrm{H}-\mathrm{NMR}\left(300 \mathrm{MHz}, \mathrm{CDCl}_{3}\right) \delta 7.19(\mathrm{t}, 2 \mathrm{H}, J=6.8 \mathrm{~Hz}), 7.12$ $(\mathrm{d}, 2 \mathrm{H}, J=7.2 \mathrm{~Hz}), 6.77(\mathrm{~d}, 2 \mathrm{H}, J=8.2 \mathrm{~Hz}), 2.61(\mathrm{t}, 2 \mathrm{H}, J=7.5 \mathrm{~Hz}), 1.61(\mathrm{~m}, 2 \mathrm{H}), 1.34-1.32(\mathrm{~m}, 4 \mathrm{H})$, $0.90(\mathrm{t}, 3 \mathrm{H}, J=6.6 \mathrm{~Hz}) ;{ }^{13} \mathrm{C}-\mathrm{NMR}\left(75 \mathrm{MHz}, \mathrm{CDCl}_{3}\right) \delta 162.9(\mathrm{dd}, J=254.1 \mathrm{~Hz}, J=5.6 \mathrm{~Hz}), 162.6$ $(\mathrm{dd}, J=255.0 \mathrm{~Hz}, J=6.4 \mathrm{~Hz}), 151.2(\mathrm{ddd}, J=251.5 \mathrm{~Hz}, J=10.6 \mathrm{~Hz}, J=4.9 \mathrm{~Hz}), 147.9$ (t, $J=9.6 \mathrm{~Hz}), 140.9(\mathrm{dt}, J=256.8 \mathrm{~Hz}, J=15.5 \mathrm{~Hz}), 125.2(\mathrm{t}, J=11.6 \mathrm{~Hz}), 118.4(\mathrm{td}), 116.2$ $(\mathrm{ddd}, J=15.4 \mathrm{~Hz}, J=7.6 \mathrm{~Hz}), 114.5(\mathrm{dd}, J=18.5 \mathrm{~Hz}, J=7.6 \mathrm{~Hz}), 111.3(\mathrm{dd}, J=17.9 \mathrm{~Hz}, J=5.2 \mathrm{~Hz})$, $102.2(\mathrm{t}, J=19.6 \mathrm{~Hz}), 98.5(\mathrm{t}, J=20.1 \mathrm{~Hz}), 97.2,95.6,80.9,77.8,36.0,31.3,30.3,22.5,13.9$; Anal. calcd for $\mathrm{C}_{27} \mathrm{H}_{17} \mathrm{~F}_{7}$ : C, 68.35; H, 3.59. Found: C, 68.38; H, 3.76.

\section{Conclusions}

In summary, we have designed and synthesized BPEBs by convenient Sonogashira cross-coupling reactions, which have different numbers of side-substitute fluorine atoms on benzene rings, and alkyl chains, ethoxyl groups, fluorine atoms and trifluoromethyl groups as the end groups. The detailed investigation of the synthesized BPEBs properties have disclosed that the melting points, clearing points, nematic phase, optical anisotropy $(\Delta n)$ and dielectric anisotropy $(\Delta \varepsilon)$ greatly depend on both the numbers of side-substitute fluorine atoms and structures of the end groups. On the basis of the obtained properties of the synthesized BPEBs, some of them have been expected to have high potential application as the compositions in blue phase liquid crystals. Therefore, a mixture of blue phase liquid crystal has been prepared with a relative wide blue phase temperature range of $8{ }^{\circ} \mathrm{C}$. The obtained results have implied that the synthesized BPEBs will certainly be important in the development of new 
types and properties of LCs. Further study on the application of BPEBs in making other new types of LCs is underway in our laboratory.

\section{Acknowledgments}

Financial support from the National High Technology Research and Development Program, China (No. 2011AA02A209) is gratefully acknowledged.

\section{Conflicts of Interest}

The authors declare no conflict of interest.

\section{References}

1. Carlton, R.J.; Hunter, J.T.; Miller, D.S.; Abbasi, R.; Mushenheim, P.C.; Tan, L.N.; Nicholas, L.; Abbott, N.L. Chemical and biological sensing using liquid crystals. Liq. Cryst. Rev. 2013, 1, 29-51.

2. Tadwee, I.; Shahi, S.; Ramteke, V.; Syed, I. Liquid crystals pharmaceutical application: A review. Int. J. Pharm. Res. Allied Sci. 2012, 1, 6-11.

3. Goodby, J.W. The nanoscale engineering of nematic liquid crystals for displays. Liq. Cryst. 2011, $38,1363-1387$.

4. Kitzerow, H.-S. Blue phases come of age: A review. Proc. SPIE 2009, doi:10.1117/12.813372.

5. Hong, H. Analysis of focal length of blue-phase liquid crystal (BPLC) cylindrical lens for the light of the various incident angles and polarizations. Liq. Cryst. 2013, 40, 450-457.

6. Yamamoto, S.-I.; Haseba, Y.; Higuchi, H.; Okumura, Y.; Kikuchi, H. Lattice plane control of liquid crystal blue phase. Liq. Cryst. 2013, 40, 639-645.

7. Li, P.; Sun, Y.; Wang, Q. A transflective and viewing angle controllable bluephase liquid crystal display. Liq. Cryst. 2013, 40, 1024-1027.

8. Tour, J.M. Molecular electronics. Synthesis and testing of components. Acc. Chem. Res. 2000, 33, 791-804.

9. Bunz, U.H.F. Poly(aryleneethynylene)s: Syntheses, properties, structures, and applications. Chem. Rev. 2000, 100, 1605-1644.

10. Levitus, M.; Schmieder, K.; Ricks, H.; Shimizu, K.D.; Bunz, U.H.F.; Garcia-Garibay, M.A. Steps to demarcate the effects of chromophore aggregation and planarization in poly(phenyleneethynylene)s. 1. Rotationally interrupted conjugation in the excited states of 1,4-bis(phenylethynyl)benzene. J. Am. Chem. Soc. 2001, 123, 4259-4265.

11. Beeby, A.; Findlay, K.; Low, P.J.; Marder, T.B. A re-evaluation of the photophysical properties of 1,4-bis(phenylethynyl)benzene: A model for poly(phenyleneethynylene). J. Am. Chem. Soc. 2002, $124,8280-8284$.

12. Schwab, P.F.H.; Smith, J.R.; Michl, J. Synthesis and properties of molecular rods. 2. Zig-zag rods. Chem. Rev. 2005, 105, 1197-1279.

13. Wu, S.-T.; Hsu, C.-S.; Shyu, K.-F. High birefringence and wide nematic range bis-tolane liquid crystals. Appl. Phys. Lett. 1999, 74, 344-346. 
14. Tanaka, T.; Sekine, C.; Ashida, T.; Ishitobi, M.; Konya, N.; Minai, M.; Fujisawa, K. Highly anisotropic molecular materials for LCD. Mol. Cryst. Liq. Cryst. 2000, 346, 209-216.

15. Long, T.M.; Swager, T.M. Triptycene-containing bis(phenylethynyl)benzene nematic liquid crystals. J. Mater. Chem. 2002, 12, 3407-3412.

16. Liao, Y.-M.; Chen, H.-L.; Hsu, C.-S.; Gauza, S.; Wu, S.-T. Synthesis and mesomorphic properties of super high birefringence isothiocyanato bistolane liquid crystals. Liq. Cryst. 2007, 34, 507-517.

17. Lydon, D.P.; Albesa-Jové, D.; Shearman, G.C.; Seddon, J.M.; Howard, J.A.K.; Marder, T.B.; Low, P.J. The synthesis and liquid crystalline behaviour of alkoxy-substituted derivatives of 1,4-bis(phenylethynyl)benzene. Liq. Cryst. 2008, 35, 119-132.

18. Dziaduszek, J.; Kula, P.; Dąbrowski, R.; Drzewiński, W.; Garbat, K.; Urban, S.; Gauza, S. General synthesis method of alkyl-alkoxy multifluorotolanes for negative high birefringence nematic mixtures. Liq. Cryst. 2012, 39, 239-247.

19. Miao, Z.-C.; Wang, D.; Zhang, Y.-M.; Jin, Z.-K.; Liu, F.; Wang, F.; Yang, H. Asymmetrical phenyldiacetylenes liquid crystalline compounds with high birefringence and characteristics of selective reflection. Liq. Cryst. 2012, 39, 1291-1296.

20. Zhang, Y.-M.; Wang, D.; Miao, Z.-C.; Jin, S.-K.; Yang, H. Novel high birefringence bistolane liquid crystals with lateral fluorosubstituent. Liq. Cryst. 2012, 39, 1330-1339.

21. Herman, J.; Dziaduszek, J.; Dąbrowski, R.; Kędzierski, J.; Kowiorski, K.; Dasari, V.S.; Dhara, S.; Kula, P. Novel high birefringent isothiocyanates based on quaterphenyl and phenylethynyltolane molecular cores. Liq. Cryst. 2013, 40, 1174-1182.

22. Yatabe, T.; Okumoto, H.; Kawanishi, Y.; Inoue, T. Charge-carrier transport in 1,4-bis(phenylethynyl)benzene derivatives exhibiting crystal mesophases. Chem. Lett. 2013, 42, 764-766.

23. Imrie, C.T.; Taylor, L. The preparation and properties of low molar mass liquid crystals possessing lateral alkyl chains. Liq. Cryst. 1989, 6, 1-10.

24. Iwata, T.; Suzuki, K.; Higuchi, H.; Kikuchi, H. A method for enlarging the Kerr constant of polymer-stabilised blue phases. Liq. Cryst. 2009, 36, 947-951.

(C) 2013 by the authors; licensee MDPI, Basel, Switzerland. This article is an open access article distributed under the terms and conditions of the Creative Commons Attribution license (http://creativecommons.org/licenses/by/3.0/). 